\title{
The VISTA Science Archive
}

\author{
N. J. G. Cross ${ }^{1}$, R. S. Collins ${ }^{1}$, R. G. Mann ${ }^{1}$, M. A. Read ${ }^{1}$, E. T. W. Sutorius ${ }^{1}$, R. P. Blake ${ }^{1}$, M. Holliman ${ }^{1}$, \\ N. C. Hambly ${ }^{1}$, J. P. Emerson ${ }^{2}$, A. Lawrence ${ }^{1}$, and K. T. Noddle ${ }^{1}$ \\ 1 Wide-Field Astronomy Unit, Institute for Astronomy, School of Physics and Astronomy, University of Edinburgh, \\ Royal Observatory, Blackford Hill, Edinburgh EH9 3HJ, UK \\ e-mail: njc@roe.ac.uk \\ 2 Astronomy Unit, School of Physics \& Astronomy, Queen Mary University of London, Mile End Road, London E1 4NS, UK
}

Received 29 April 2012 / Accepted 9 October 2012

\section{ABSTRACT}

\begin{abstract}
We describe the VISTA Science Archive (VSA) and its first public release of data from five of the six VISTA public surveys. The VSA exists to support the VISTA surveys through their lifecycle: the VISTA Public Survey consortia can use it during their quality control assessment of survey data products before submission to the ESO Science Archive Facility (ESO SAF); it supports their exploitation of survey data prior to its publication through the ESO SAF; and, subsequently, it provides the wider community with survey science exploitation tools that complement the data product repository functionality of the ESO SAF.

This paper has been written in conjunction with the first public release of public survey data through the VSA and is designed to help its users understand the data products available and how the functionality of the VSA supports their varied science goals. We describe the design of the database and outline the database-driven curation processes that take data from nightly pipeline-processed and calibrated FITS files to create science-ready survey datasets. Much of this design, and the codebase implementing it, derives from our earlier WFCAM Science Archive (WSA), so this paper concentrates on the VISTA-specific aspects and on improvements made to the system in the light of experience gained in operating the WSA.
\end{abstract}

Key words. surveys - catalogs - astronomical databases: miscellaneous

\section{Introduction}

One of the clearest trends in observational astronomy over the past two decades has been the rise in the importance of systematic sky surveys. When coupled with good archives, sky surveys provide homogeneous, re-usable data products facilitating a range of research programmes and, in particular, enabling the large-scale statistical analyses required for many of the most important science goals in modern astronomy.

Probably the most prominent of this generation of surveys has been the Sloan Digital Sky Survey (SDSS; York et al. 2000) and its SkyServer archive system (Szalay et al. 2002) demonstrated the power of a survey archive based on a relational data base management system (RDBMS). Such systems can offer astronomers the ability to pose powerful analytical queries in structured query language (SQL) against seamless, survey-wide source catalogues, thereby enabling survey science that would be impossibly cumbersome for an astronomer provided with nothing more than a repository of matching image and catalogue files for each of the thousands of pointings making up the survey dataset.

The success of the SDSS SkyServer was a strong influence on the design of the archive component of the VISTA data flow system (VDFS; Emerson et al. 2004). VISTA, the Visible and InfraRed Survey Telescope for Astronomy, is described by Emerson et al. (2006). VDFS was designed as a two-phase project, with an initial goal of supporting near-infrared surveys to be conducted with the Wide Field CAMera (WFCAM; Casali et al. 2007) on the UK InfraRed Telescope (UKIRT) and an ultimate objective of supporting surveys with VISTA. Within VDFS, the Cambridge Astronomy Survey Unit (CASU) run a night-by-night data processing pipeline, with the Wide-Field Astronomy Unit (WFAU) in Edinburgh generating further data products and providing science archive facilities.

The first-generation VDFS archive - the WFCAM Science Archive (WSA) - is described by Hambly et al. (2008) and serves catalogue and image data from the UK Infrared Deep Sky Survey (UKIDSS; Lawrence et al. 2007), as well as other, P.I.-mode, data taken with WFCAM. More than 1000 users are registered for authenticated access to proprietary UKIDSS data through the WSA, and it supports anonymous access by a larger community once the data are public. The phased approach adopted within the VDFS ensured that the design and development of the WSA progressed with scalability to the larger data volumes of VISTA kept explicitly in mind, along with the likely scientific usage patterns of the VISTA surveys. For example, Cross et al. (2009) describe the enhancements made to the WSA database schema to support time-series analysis of multiepoch data, which was prototyped using observations from the UKIDSS Deep Extragalactic Survey, but motivated by the requirements for supporting variability analyses with VISTA.

The initial scientific programme for VISTA is mostly focussed on six ESO Public Surveys which deliver reduced images and derived catalogue data products to the ESO Science Archive Facility (SAF). Five of the six Public Survey consortia (see Sect. 2), use the VDFS for the generation of these data products and employ the VISTA Science Archive (VSA) to manage their data, both for quality assurance analysis and preliminary exploitation prior to submission to the ESO SAF and, following its publication there, to provide the wider community with sophisticated science archive capabilities that complement the data product repository functionality of the ESO SAF. 


\subsection{Summary of earlier VDFS work}

Since the design of the VSA is derived so directly from that of the well-used WSA, we strongly urge readers who are unfamiliar with the VDFS science archives to read the existing papers on the WSA (Hambly et al. 2008; Cross et al. 2009), as well as the comprehensive online documentation provided on the VSA website $^{1}$, in conjunction with this paper. However, we will summarise the main points of these papers which are relevant to the VSA in this section.

VISTA and UKIRT/WFCAM take very similar basic data, which consists of images obtained by a wide-field near infrared imaging array on a $\sim 4 \mathrm{~m}$ telescope. The two cameras utilise large format $2048 \times 2048$ pixel infrared detectors: sixteen 0.34 arcsec pixel $^{-1}$ in the case of VISTA, and four 0.4 arcsec pixel $^{-1}$ in the case of WFCAM. Images are taken in 5 broad-band filters $\left(Z, Y, J, H, K_{\mathrm{S}} / K\right)$ and several narrow-band filters, within the wavelength range $0.8<\lambda<2.5 \mu \mathrm{m}$, for a range of large surveys and smaller P.I. programmes. Large areas can be covered by repeating a basic pattern of images, a square pattern of 4 adjacent pawprints in the case of WFCAM.

Observing time is divided between large surveys (UKIDSS and the Campaigns on UKIRT/WFCAM) and smaller PI-led programmes (time awarded by a telescope allocation committee), service mode observations for very small projects and special projects like director's discretionary time projects and calibration work. WFAU ingest all of these data into the WSA but the key design features were driven by the requirements for the main surveys: the UKIDSS surveys and the then future VISTA Public Surveys.

In VDFS, a single exposure, reduced science frame is designated as a normal frame. These frames can be stacked (coadded to increase the signal-to-noise) together with small offsets in position (a jitter pattern) to reduce the effects of bad pixels: the resulting image frame is designated a stack frame. The pixel size of WFCAM images is $0.4^{\prime \prime}$, but the seeing sometimes is as good as $0.5^{\prime \prime}$, so the images can be undersampled. To produce critically sampled images in the best seeing conditions, a technique called micro-stepping was used, where a series of images are interleaved; adjacent frames that are offset by half or a third of a pixel, (in reality a small integer offset is added too to reduce the affects of bad pixels) in the $x$ and $y$ directions, to create a $2 \times 2$ (leav) image with $0.2^{\prime \prime}$ (or $3 \times 3$ with $0.133^{\prime \prime}$ ) pixels. Several leav frames with different jitter positions are stacked together, the resulting image frame is designated a leavstack frame.

A stack (leav or otherwise) is the fundamental science image, from which catalogues are extracted. If there are multiple epochs in the same filter and pointing, a deepstack or deepleavstack may be created to find fainter sources. All of these science frames have associated confidence (conf) images that contain the relative weighting of each pixel. This includes bad pixel masking, the effects of jittering, seeing and exposure time weighting.

At WFAU, for the purposes of archiving, UKIDSS as a whole was referred to as a survey while the different parts of it: the Large Angular Survey (LAS), the Galactic Plane Survey (GPS), the Deep Extragalactic Survey (DXS) etc. were referred to as programmes and PI-led programmes were referred to as nonsurvey programmes. Since all UKIDSS programmes were released together and there was a management structure covering the whole of UKIDSS, the distinction between survey and programme was useful and clear. UKIRT divide observations into projects which have their own identifiers. We assign projects to

\footnotetext{
1 http://surveys.roe.ac.uk/vsa
}

programmes based on the metadata identifiers, e.g. in the header, the PROJECT keyword u/ukidss/gcs5 is UKIDSS GCS project observing set number 5 and is assigned to programmeID $=103$ in the WSA.

The data in the WSA are stored in a RDBMS, which stores data in a set of related tables. In an RDBMS, missing values can be handled in a couple of ways: by setting them as NULL, or giving them a default value. Setting them as NULL has the advantage that it is clear that data is missing, but has the disadvantage that all queries have to consider NULL values. For example, if you are querying for objects with a certain colour range, $-1.0<(J-H)<1.0$, it would be necessary to include a constraint "and $(J-H)$ is not null", which would occur if one or other of the two images had not yet been observed or the object was too faint in the $J$ or $H$ band for a detection. Our solution is to use default values, but our standard default values are large negative values, that are outside the normal range of most physical values. The default values for each column are given in the schema browser, see below. All of the columns in all of the tables are not null, i.e. they have at least a default value.

The pixel data from images is not stored directly in the RDBMS as binary large objects (BLOBs), but they are stored as flat files in multi-extension FITS (MEF; a FITS file, Pence et al. 2010, with a primary which contains metadata about the whole file, and secondary extensions with binary data from each detector and a header with detector relevent attributes) format, and the paths are stored in the archive, along with all of the metadata. The databases are self describing, since all the information about the surveys is also stored in the database, in curation tables, which are database tables that are designed principally to aid the curation of each programme, see Sect. 3.1.5.

The design of the relational database should capture the inherent structure of the data stored. For instance the image metadata can be divided into different groupings, e.g. metadata related to the whole MEF, such as observation time, filter, project, PI, airmass and metadata related to each detector extension, such as sky level, zeropoint, seeing. When designing a relational database, we capture the structure in an entity-relation model (ERM), see Fig. 4 for an example. An ERM contains entities (shown as boxes with rounded corners) which represent a collection of related data, e.g. primary header data from each FITS image or sources in a merged filter catalogue. The relationship between entities is represented by lines between them which are mandatory (solid line), optional (dotted) and can be one-toone (a single line), one-to-many (a single line that branches into three, similar to a crow's foot) or many-to-many (three lines converge to a single line that branches into three). If the two tables share the same primary key a perpendicular line is added across the main line. The basic ERMs for the WSA (which are relevant for the VSA) are shown in Hambly et al. (2008) and we show the multi-epoch ERMs in Cross et al. (2009).

While the database models presented so far could be implemented in any RDBMS, the WSA and VSA are implemented in a commercial software product, Microsoft SQL Server, which is suitable for medium to large scale databases. This was also the choice that the Sloan Digital Sky Survey team made, which heavily influenced our decision. When we implement the data model the ERMs are converted into a schema, a set of database objects. Most of these objects are tables, with entities in the ERM mapping to tables in the schema. The tables hold all the data and can be queried via the user interface applications.

The WSA provides a schema browser, which contains all the information about all of the tables in each release. The left hand side gives the user a list of surveys and releases and then the 
tables, functions and views associated with each one. Selecting a table gives a description of the table and then the list of attributes in the table. Each attribute has the name, type (e.g. int, real, float), length (in bytes), units, description, default value and unified content descriptor. Each table has a primary key, which is a single attribute or a combination of attributes in the table that between them can cover all unique entries and must be unique. For instance, the Multiframe table contains entries for each multi-extension FITS image, so the primary key is the multiframeID, whereas in the lasDetection table, entries are objects extracted from each extension of a FITS image, and within each extraction are given a sequence number, so the primary key is (multiframeID, extNum, seqNum). Any constraints with respect to other tables are listed at the top and all attributes which are indexed for fast searches are highlighted. The primary key attributes are indexed automatically. Some attributes require a more detailed description, and these are linked to a glossary and have a symbol like a book beside the attribute name, which can be clicked on.

Other database objects include Views and Functions. Views are selections of data from tables already in the database. They can be a subset from one table or a superset of many, and are queried in the same way as tables, but no extra data is stored. These are used, for instance, in the WSA when we give a subset of the source tables for some UKIDSS surveys which have data taken in all filters. Functions take inputs and do specific calculations. We have some which do spherical astronomy calculations, give expected magnitude limits, convert RA and Dec to a sexagesimal string and give names for objects in the IAU convention.

\subsection{Outline of paper}

This paper describes the VSA and its first public release of data from the five VDFS-supported Public Surveys. In Sect. 2 we discuss the VISTA telescope and the Public Surveys and compare to UKIRT-WFCAM, focussing on the essential differences that affect the VSA. In Sect. 3, we provide an overview of the VSA, discussing the table structure before we compare the VSA to the WSA in Sect. 4. We discuss changes to the image metadata, the catalogue parameters and the infrastructure in Sects. 5-7 in the VSA compared to the WSA and new features that are common to both in Sect. 8. Section 9 provides examples of some of the different types of science queries that the VSA supports, while Sect. 10 provides details of the contents of the first VSA releases of the five VDFS-supported VISTA Public Surveys. We summarise this paper and discuss future work in Sect. 11, while several appendices provide technical details supplementing the main body of the paper.

\section{Overview of VISTA and its public surveys}

VISTA (Emerson et al. 2006) is currently the fastest nearinfrared survey telescope, with an étendue (area times instantaneous field-of-view) of approximately $6.5 \mathrm{~m}^{2} \mathrm{deg}^{2}$. It has a $4 \mathrm{~m} \mathrm{f} / 1$ primary mirror, and a $1.2 \mathrm{~m}$ secondary giving it a 1.65 degree diameter field-of-view (see Emerson \& Sutherland 2010a,b). The VISTA Infra Red CAMera (VIRCAM; Dalton et al. 2010), has $162048 \times 2048$ pixel non-buttable Raytheon VIRGO HgCdTe detectors and has a quantum efficiency $>80 \%$ between $0.9 \mu \mathrm{m}$ and $2.4 \mu \mathrm{m}$. The pixel scale is $0.34^{\prime \prime}$ and the instantaneously sampled field-of-view is 0.6 sq. deg (see Fig. 1). Compared to its nearest counterpart, the United Kingdom Infra Red Telescope with its Wide Field CAMera (UKIRT-WFCAM; Casali et al. 2007), the survey speed of VISTA is $\sim 6$ times faster, having twice the sensitivity - increased throughput for a similar sized telescope - and 3 times the area per pointing.

ESO's Science Verification for VISTA started at the end of 2009 and the main science programme commenced in early 2010. VISTA's programme initially focuses on six ESO Public Surveys ${ }^{2}$, nicely complementing the sub-surveys of UKIDSS in the northern sky.

These six surveys are:

- VHS: the VISTA Hemisphere Survey ${ }^{3}$;

- VVV: the VISTA Variables in Via Lactea ${ }^{4}$ (Saito et al. 2012);

- VMC: the VISTA Magellanic Cloud $\operatorname{survey}^{5}$ (Cioni et al. 2011);

- VIKING: the VISTA Kilo-degree INfrared survey for Galaxies (Findlay et al. 2012);

- VIDEO: the VISTA Deep Extragalactic Objects survey ${ }^{6}$ (Jarvis et al. 2012);

- UltraVISTA ${ }^{7}$ (McCracken et al. 2012).

These surveys have a "wedding cake" arrangement of galactic/extragalactic surveys (VHS, VIKING, VIDEO, UltraVISTA) with different depth/area combinations and two specialised stellar astronomy programmes (VVV, VMC), much like the UKIDSS surveys. The five surveys supported by the VDFS are VHS, VVV, VMC, VIKING and VIDEO. UltraVISTA makes use of the CASU pipeline products, but is not currently archiving its data in the VSA.

VISTA data is calibrated on the natural VISTA photometric system (see Hodgkin et al., in prep.). All magnitudes (unless designated as $A B$ mag) are on this Vega mag system.

\subsection{Differences in the telescope and instrument between VISTA and WFCAM}

For detailed descriptions of VISTA and WFCAM, see Emerson et al. (2006) and Casali et al. (2007) respectively. In this section we will just discuss the salient differences which affect the VSA design compared to the WSA.

\subsubsection{The VISTA focal plane: pawprints and tiles}

VISTA is significantly different from UKIRT/WFCAM in several important aspects, which affect image processing and subsequent archive operations. The most significant differences of VISTA to UKIRT/WFCAM are the arrangement of the focal plane and the ability VISTA's alt-azimuth mount provides to observe the same piece of sky in any orientation with respect to the focal plane.

VISTA has $162 \mathrm{k} \times 2 \mathrm{k}$ Raytheon VIRGO detectors arranged in a pawprint with detectors spaced $90 \%\left(10.4^{\prime}\right)$ of a detector apart in the $X$-direction and $42.5 \%\left(4.9^{\prime}\right)$ apart in the $Y$-direction (see Fig. 1) whereas WFCAM has $42 \mathrm{k} \times 2 \mathrm{k}$ Hawaii 2 detectors arranged in a pawprint (see Fig. 2) with the same spacing of $94 \%$ $\left(12.8^{\prime}\right)$ in each direction.

The VISTA basic filled survey area is a tile made up of six pawprints, three in the $Y$-direction separated by 0.475 of a detector, and two in the $X$-direction separated by 0.95 of a detector.

\footnotetext{
2 http://www .eso.org/public/teles-instr/ surveytelescopes/vista/surveys.html

3 http://www . ast. cam.ac.uk/ rgm/vhs/

4 http://vvvsurvey.org

5 http://star.herts.ac.uk/ mcioni/vmc/

6 http://star-www.herts.ac.uk/ mjarvis/video/

7 http://www.strw. leidenuniv.nl/ ultravista/
} 


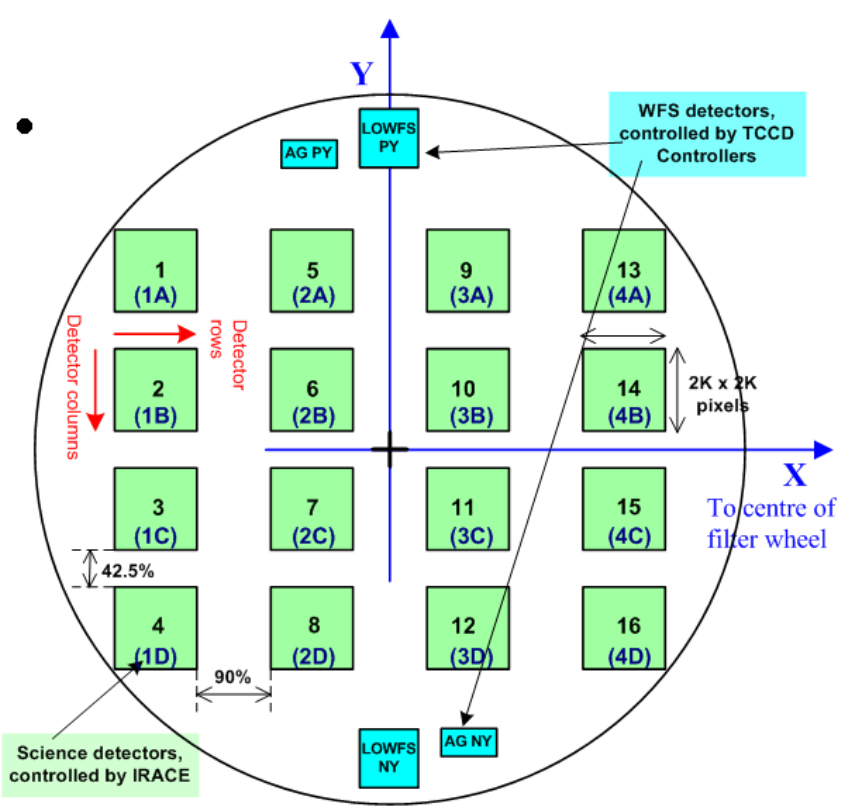

Fig. 1. VISTA focal plane showing $162 \mathrm{k} \times 2 \mathrm{k}$ detectors with $90 \%$ spacing in the $x$-direction and $42.5 \%$ in the $y$-direction. There are also two auto-guider (AG) and two low-order wave-front sensor (LOWFS) detectors.
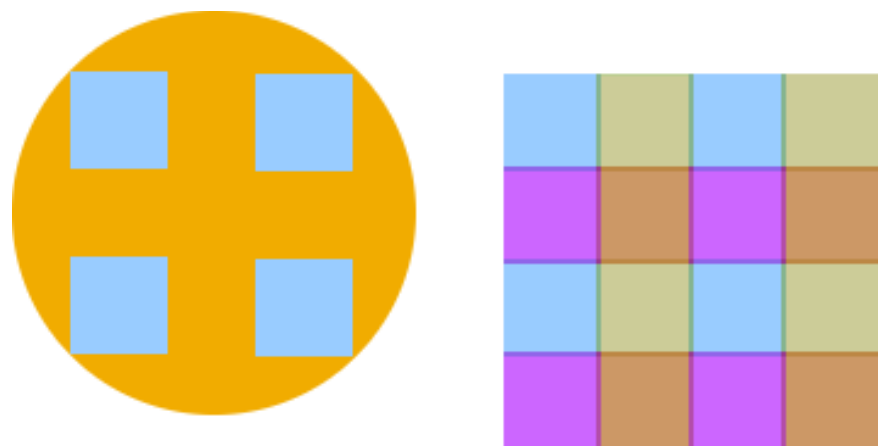

Fig. 2. Areas of sky can be efficiently surveyed by arranging 4 WFCAM pawprints (left) into the arrangement on the right. Each colour in the right hand image represents a different pawprint. There is a small amount of overlap at each edge.

Except at two strips with just a single exposure, this tile has between twice and six times the exposure time of each pawprint at every pixel with a mode of two exposures (see Fig. 3). There are two possible ways to achieve a required uniform minimum (two pawprints contributing) depth across a multi-tile survey. The most efficient in terms of observing time is for successive tiles to overlap the strips at top and bottom, coadding the data from the two separate tiles, and each will have an area of 1.636 sq. deg covered at least twice. However reaching the full depth by coadding these two strips can be complicated by varying sky conditions if the adjacent tiles are not observed under the same conditions (e.g. different PSFs and sky conditions). Indeed the same two effects can be a problem in making a tile from six pawprints (depending on how rapidly the seeing varies between pawprints). The other less efficient, but simpler, way to achieve constant depth across a multi-tile survey is to butt together regions of tiles that have reached the minimum double exposure, ignoring the singly exposed strips resulting in an area of $1.501 \mathrm{sq}$. deg covered at least twice.

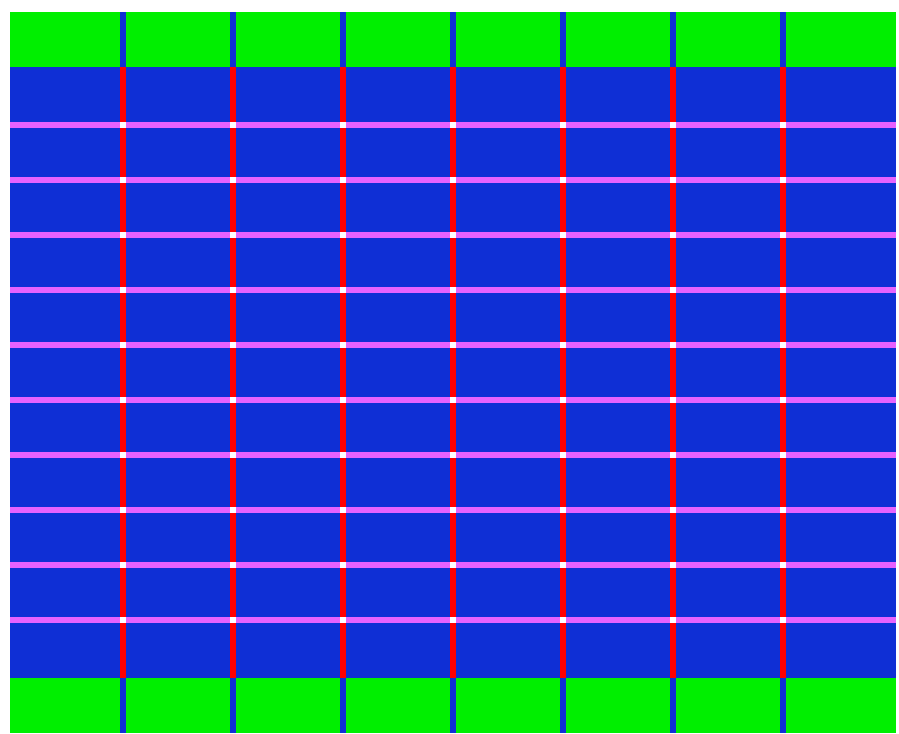

Fig.3. An exposure map of a VISTA tile. The green strips at top and bottom have a single exposure. The majority of the area (blue) has two exposures, the pink has 3 exposures, the red 4 and the white 6 . The doubly or more exposed area is $1.501 \mathrm{sq}$. deg. The singly exposed green strips at top and bottom of the plot are each $1.475 \mathrm{deg} \times 0.092 \mathrm{deg}=$ 0.135 sq. deg and can be overlapped by corresponding areas from adjacent tiles for many surveys. Assigning one of the two 0.092 deg overlap (top and bottom) to each of the adjacent tiles involved in an overlap, means that each tile, when part of a filled larger area, covers $(1.017+$ $0.092) \times 1.475=1.636$ sq. deg at least twice.

In the case of WFCAM four pawprints are required to make a filled tile, and everything gets a single exposure and there are no edge strips, so large contiguous regions of the sky can be surveyed by simply overlapping subsequent pawprints as shown in Fig. 2. We note that in common with WFCAM, "microstepping" is possible with VISTA, but it is not usually necessary since the pixel size on VISTA is smaller than WFCAM and the typical seeing at Cerro Paranal is slightly worse than Mauna Kea, so most images are critically sampled. Moreover, its use is not recommended by ESO and the VISTA Public Surveys do not use the technique.

\subsubsection{Merging VISTA pawprints into tiles}

The processing of raw pawprint frames into calibrated images is done by the VDFS pipeline at CASU on a nightly basis ${ }^{8}$ and includes combining the pawprints into tiles. These pawprints and tiles are ingested into the VSA without any additional image processing. If any pointing is observed only once in a given filter in the survey design, the nightly pipeline-processed catalogue product is used to generate the merged Source (see Sect. 3.1) table. For deeper surveys, however, stacking tiles observed multiple times typically involves observations over several different nights, and multi-night products are not the responsibility of CASU. WFAU creates tiles from multiple nights of data by first stacking the separate component pawprints and then combining the six stacked pawprints into a tile. Stacking the tiles rather than the component pawprints is very firmly not recommended.

While tiling pawprints together to form a tile is quite standard when working with visible images (Fruchter \& Hook 2002), ground-based infrared sky subtraction is difficult because the sky

\footnotetext{
8 http://casu.ast. cam.ac.uk/surveys-projects/vista/ technical
} 
is so much brighter than in the optical and dominates the flux of most objects. Furthermore, the VISTA camera's distortion across the field-of-view and the larger variation in both the sky and the point-spread function (PSF) within the duration of the observations needed to create a single tile make it necessary to do additional processing (Lewis et al. 2010). Tiles are processed using the following procedures ${ }^{9}$ :

- stack all components of each pawprint to reach the intended signal-to-noise while removing bad pixels by taking the clipped median flux value. Each component is shifted by a different number of pixels in the $X$ and $Y$ directions as defined by the jitter pattern so that bad pixels or columns appear in different positions in each component;

- extract the catalogue from each pawprint. This detects collections (at least 4) of pixels which are all brighter than the background by more that 1.5 times the sky noise. Overlapping objects are deblended;

- recalculate the world coordinate system (WCS) of each pawprint, by comparing stars in the catalogue to the Two Micron All Sky Survey (2MASS) catalogue (Skrutskie et al. 2006);

- calculate the photometric zero-points (VISTA system) of each detector in each pawprint and update the headers. For observing block processing this is done compared to 2MASS, using the colour equations calculated by CASU and whichever stars of the correct colours are in common with 2MASS and the VISTA image in question. For deep stacks and tiles, individual stars are compared to the component frames which have already been calibrated using 2MASS. The stars in the components and the deep stack are all on the same VISTA system, so a direct comparison is possible;

- filter each pawprint to smooth out large scale variations ( $>30^{\prime \prime}$ in the background), see Irwin (2010) for details;

- mosaic the 6 unfiltered pawprints into a tile, to produce a tile with large scale features present. Mosaicing adjusts all 96 components (16 detectors in each pawprint) to the same level and drizzles them on to a single tangent-plane projection image;

- mosaic all the filtered pawprints to produce a tile without large-scale background features;

- extract the catalogue from the filtered tile;

- recalculate the WCS of the tile;

- calculate the photometric zero-point (VISTA system) of the tile catalogue;

- "grout" the tile catalogue to find the correct PSF in each region of the tile and the correct offset for the Modified Julian Date. The grouting procedure tracks the variable flux within the first seven pre-defined circular apertures, with radii of $0.5^{\prime \prime}, \frac{1}{\sqrt{2}}{ }^{\prime \prime}, 1^{\prime \prime}, \sqrt{2}^{\prime \prime}, 2^{\prime \prime}, 2 \sqrt{2}^{\prime \prime}$ and $4^{\prime \prime}$. Differential aperture corrections (i.e. the difference between the flux in the aperture and the total flux for a point source) are calculated for each detector in each pawprint that composes the tile. The fluxes in these 7 apertures are then recalculated, although larger aperture fluxes and other fluxes, such as the Petrosian flux are not corrected. These larger apertures will be only marginally affected by the seeing variations;

- reclassify the stars and galaxies in the tile catalogue. Classification originally occurs as part of the extraction process and uses the curve of growth to calculate a stellarness-of-profile statistic and classification (galaxy 1, star -1 , noise 0 , saturated -9 , probable star -2 , or probable

\footnotetext{
9 http://casu.ast.cam.ac.uk/surveys-projects/vista/ technical/tiles gives more details of the algorithms.
}

galaxy -3). The different PSFs in each pawprint when combined can give misleading classifications, so the classification code takes the grouting information about each pawprint and re-estimates the stellarness-of-profile statistic and classification;

- recalculate the photometric zero-point (VISTA system) of the tile catalogue;

- remove any temporary files, such as the filtered pawprints.

Unsurprisingly the filtering also makes it impossible to produce accurate catalogue values for large extended sources, e.g. nearby galaxies or Galactic nebulae. The filtering scale used for the main VISTA Public Surveys removes structure on scales larger than 30". This is a similar scale to the local background scale length (22") which would in any case limit the accuracy of any photometry of larger objects.

\subsubsection{Active optics}

The quality of VISTA images is maintained, as it observes at different elevations and its temperature changes, by updating the position of the secondary mirror, with corrections derived from look up tables and the low order wave front sensors. If a correction has not been applied recently enough, or it is bad for some reason, the image quality can be degraded. Some images show evidence for such problems, contributing, along with seeing variations between pawprints, and PSF variation across the field when in perfect alignment, to a greater variety of PSFs than in the case of WFCAM.

\subsubsection{Telescope mounts}

VISTA is an alt-azimuth mounted telescope, whereas UKIRT has an equatorial mount, so the focal plane in WFCAM remains in the same equatorial orientation, but the VISTA focal plane must rotate with respect to the telescope to keep the same orientation on the sky during an exposure. Since the focal plane can rotate, orientation is an additional degree of freedom. Different programmes can choose the orientation that best accommodates their survey design. Given the complex processing of image and catalogue data (see previous subsection), stacking two images at very different orientations was considered inadvisable. Thus we group data based on orientation as well as position/filter when stacking and tiling and to do this we have added an extra column, posAngle to the RequiredStack (see Sect. 3.1) table. This is the orientation of the image $x$-axis to the N-S line. This means that if images in the same programme lie in the same position and filter but have very different orientations they will be processed separately. As a default, we have been using a tolerance of $15 \mathrm{deg}$, but it can be set programme by programme. In practice this situation has only occurred once in the Public Surveys: the VVV team had a small amount of data in the Science Verification stage which was orientated along equatorial RA axis, and later data in the same region of sky which was orientated along the Galactic longitude axis, and so the tiles are orientated at $60 \mathrm{deg}$ to each other.

\section{Overview of the VSA}

\subsection{The basic structure of the VSA}

WFAU receive VISTA data as multi-extension FITS (MEF; Pence et al. 2010) files: primarily these come from the CASU 
pipeline, which processes the data by Observing Block (OB), but WFAU can also handle "external" FITS images made outside VDFS, such as those produced by the survey consortia, as is currently done with deep VIDEO mosaics, with WFAU merely modifying the headers and file names prior to ingestion so that the ingestion code can correctly handle them. The MEFs contain either images or catalogues of objects extracted from them. Each MEF consists of a primary header, containing metadata relevant to the whole observation of the associated pawprint, and one extension for each detector of the pawprint, containing metadata relevant to this detector as well as the binary data (image or catalogue) associated with this detector. In VSA parlance the content of an MEF image file is a multiframe (consisting of images - frames - for all detectors) and a detection is an object extracted from a single image in a single filter. The metadata headers from these files are loaded into tables within an RDBMS, as are the catalogue tabular data. The header data consists of multiple cards, each no longer than 80 characters, which contains the keywords with their values and descriptions. A standard keyword has a length of 8 characters, but longer, more descriptive ones, or even keywords consisting of multiple words can be used by preceding them with "HIERARCH". HIERARCH keywords originating at ESO start with "HIERARCH ESO".

Depending on the survey, further data products may be generated in a database-driven manner. For example, repeated observations of the same field in the same filter may be stacked to create deeper images, from which catalogues are then extracted, while information from multiple different single-filter catalogues are merged to create catalogues of sources, which are objects described by attributes in several filters. The metadata from the additional image data products are ingested into database tables, as are derived catalogues, while the images themselves are stored in FITS format on disk. Further information may then be derived from the database tables and stored in new tables: e.g. variability information may be derived from multi-epoch data, following the synoptic data model of Cross et al. (2009). The VSA comprises, therefore, a set of tables within an RDBMS, a collection of FITS files stored on disk and the interfaces that allow users to access these data.

Whole image files may be selected for download from the archive through web forms or via SQL queries on the image metadata tables, while image cut-outs may be created from these using a different web form. Other web forms exist to provide a basic level of access to the catalogue data, but the real power of the VSA comes from the ability to query RDBMS tables using SQL. To do this requires knowledge of the VSA database schema. The online VSA schema browser ${ }^{10}$ provides detailed descriptions of every column in the hundreds of database tables in the VSA, but we summarise the five main table classes in the remainder of this section.

Throughout this paper, we use a fixed-width font to refer to VSA database tables: e.g. Multiframe or vvvSource. Many of the tables are set up for individual programmes (e.g. an individual Public Survey), such as vvvSource for the VVV, and videoSource for VIDEO. When we are discussing generic properties of "Source" tables, we will abbreviate them as Source, rather than using programme-specific names. Individual columns within tables are referred to using a bold font: e.g. multiframeID or aperMag3.

\footnotetext{
${ }^{10}$ http://surveys.roe.ac.uk/vsa/www/vsa browser.html
}

\subsubsection{Metadata tables}

The following tables record metadata about images:

- Multiframe: this contains the main primary header keywords from the VISTA images and some additional derived quantities that are calculated for each multi-extension image.

- MultiframeDetector: this contains the main extension header keywords from the VISTA images except for astrometry related keywords and some additional derived quantities that are calculated for each detector.

- CurrentAstrometry: this contains the astrometric related extension keywords and some additional derived astrometric quantities.

- MultiframeEsoKeys: this contains subsidiary primary header keywords that are stored in the hierarchical ESO format (HIERARCH ESO).

- MultiframeDetectorEsoKeys: this contains subsidiary extension header keywords that are stored in the hierarchical ESO format.

- AstrometricInfo: this includes additional derived astrometric properties, for $\mathrm{OB}$ frames (i.e. those created in a single observing block) used in multi-epoch surveys: the halfspaces (see Sect. 8.1.1) for each edge of each frame and small offsets that can be applied to the frames to improve the astrometric precision.

\subsubsection{Catalogue data tables}

The following are the catalogue data tables used in the VSA. There is a different table for each programme, so they will each start with the programme acronym:

- Detection: this contains the extracted sources for each science stack detector frame in MultiframeDetector (individual frame in a multiframe): the raw extraction attributes from the original FITS table, the calibrated positions and magnitudes and a few other derived quantities.

- Source: this is a merged filter catalogue from the deepest images in each pointing, and is made "seamless" (Hambly et al. 2008) to allow the user to find the most complete set of unique sources in the programme.

- SynopticSource: this is a merged filter catalogue made from detections in contemporaneous images. This is useful if colours of variable stars are needed. Only those programmes designed to have contemporaneous colours will have a SynopticSource table.

- Variability: this contains statistics for the light-curves of sources in multi-epoch programmes, allowing selection of variables based on different statistical quantities. VarFrameSetInfo is a useful supporting table for Variability and includes the fitted noise functions for each pointing.

\subsubsection{Linking tables}

The following are tables that link different types of catalogue data or metadata:

- Mergelog: for each pointing this lists the image frames in each filter from which the extracted detections were merged together to form the sources in the Source table. These are the deepest frames in each pointing.

- SynopticMergeLog: for each pointing at each epoch, this lists the image frames in each filter from which the extracted 
detections were merged together to form the sources in the SynopticSource table.

- BestMatch tables: these link the sources in the Source table to each epoch detection in multi-epoch surveys, to match epochs for light-curves. There are SourceXSynopticSourceBestMatch tables for "contemporaneous" filter data and SourceXDetectionBestMatch tables otherwise.

- Neighbour tables: these are simple tables containing all sources from the master table matching sources from the slave table within a specified radius. These can used for multiple purposes, such as to link with external surveys, e.g. vhsSourceXDR7Photo0bjAll links the VHS Source table to the Sloan Digital Sky Survey Data Release 7 PhotoObjAll table.

- TilePawPrints: this links tile image detections to the detections from pawprint images that make up the tile.

- Provenance: this links image frames to their components, e.g. a deep stack to each epoch stack frame that went into it, or an epoch stack frame to the raw images.

- ProgrammeFrame: this assigns image data to a programme and the programme requirements and is very important for programme curation. The same frame could be used in multiple programmes, for instance different PI programmes with the same PI in different semesters or an all hemisphere release containing data from VHS, VVV, VIKING and VMC.

\subsubsection{External catalogues}

The scientific goals of surveys tend to require external data (e.g. from surveys on other telescopes/instruments at different parts of the electromagnetic spectrum), in addition to data from VISTA itself. To support those analyses, the VSA contains copies of catalogues from a number of external surveys, which are listed in the online schema browser. The list of these is updated in response to requests from the survey consortia, and new crossmatch neighbour tables are added for different programmes, as these external surveys become available. The online documentation explains how these cross-neighbour tables can be used to perform effective cross-catalogue queries.

\subsubsection{Curation tables}

As mentioned above, the operations of the VSA are databasedriven once the original MEFs have been ingested, with processing steps and data product provenance recorded automatically in the database. The VSA contains, therefore, a large number of tables that drive, and are derived from, these curation tasks. Many of these are only of relevance to the VSA operations team, the following list do contain some pertinent information for users of the VSA:

- Programme: basic programme information. This includes the programme dependent information used to create the SQL schema which drives most curation tasks.

- RequiredTile: the current expected tile product pointings and matching tolerance. In the case of VIDEO, for which we ingest mosaics provided by the survey team, the relevant table is RequiredMosaic.

- RequiredNeighbours: lists which neighbour tables that join surveys have been created and what are the matching radii.
- PreviousMFDZP: the photometric calibration history of each image extension. MFDZP is a contraction of MultiframeDetector zeropoint.

\section{Differences between WFCAM and VISTA Science Archives}

While the design of the WSA was developed with ultimate application to VISTA in mind, there are some differences between the WSA and VSA structures.

\subsection{Tile and pawprint information in the VSA}

In the VSA, we store catalogues from both the pawprints and tiles in the detection tables for each survey (e.g. vhsDetection for the VHS survey). The tile catalogues are needed to produce uniform catalogues to the full depth of each survey. However, the astrometric solution in tile catalogues is not quite as good as that in pawprint catalogues because the distortion is not as well represented by the tangent plane (TAN) projection which tiles are projected onto, as it is in the zenithal polynomial projection (ZPN) that can be used for the pawprints, (see Calabretta \& Greisen 2002). Saturated stars also have better photometry in the pawprint catalogues. Producing pawprint catalogues does not add any additional overhead, since they must be produced as part of the production of tiles to allow the pawprints to be aligned correctly before mosaicking.

Having both tiles and pawprints has created the need for multiple layers of products and more complicated archive curation infrastructure (see Sect. 7) to keep track of these and allow them to be used together. It also means that stack requirements need an additional constraint, the offset position. Each stack that goes into a tile has a different offset position, $0-5$, which is a function of the difference (in arcseconds) of the centre (optical axis) of the pawprint from the centre of the tile. These offsets are stored as offsetX, offsetY in the Multiframe table. The offset position is not the same as the offsetID in Multiframe, which is simply the order that the offset was observed in and may differ in relative position on the tile from one epoch to the next (i.e. the order in which the pawprints are executed can be chosen by the observer but the relative positions of the 6 pawprints are fixed in the OBs currently allowed). However, the offsetPos always refers to the same part of the tile. There can be considerable overlap between two pawprints, from different parts of two different tiles, but they will not be stacked together.

\subsubsection{The Tile-PawPrint matching tables}

To link the tile and pawprint catalogues together, we have created two extra tables: TileSet and TilePawPrints, which match each detection in a tile catalogue with detections at the same position in the pawprint catalogues. These tables are survey specific, so VHS, which has detections in vhsDetection will have tables vhsTileSet and vhsTilePawPrints to link the tile and pawprint catalogues. TileSet and TilePawPrints are designed along the lines of SynopticMergeLog and SynopticSource (Cross et al. 2009): TileSet links the frames together using the multiframe identifiers for the tile and pawprints, and TilePawPrints links the detections using the extension numbers (i.e. detector number) and sequence numbers (i.e. order that object was extracted in the frame). The TilePawPrints table is deliberately as narrow as possible, and simply includes the necessary linking information, with no 


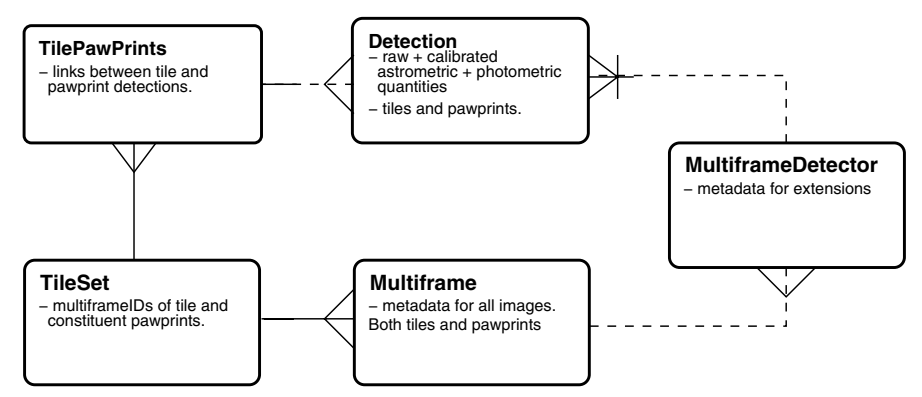

Fig. 4. ERM for the tile-pawprint linking tables. TileSet links the 7 multiframes ( 1 tile and its 6 constituent pawprints) in Multiframe. Each row in TileSet links to many associated rows in the TilePawPrints table, each of which refers to a different object. Each of these objects has between 1 and 7 measurements (detections) in the Detection table. The complication comes from TileSet containing a mix of tile and pawprint frames which cover different areas on the sky. The tile has a single extension and the pawprints have 16 extensions in MultiframeDetector.

additional attributes such as magnitudes, since it is expected that it will always be used to link, and could be used with a whole variety of attributes. By not including magnitudes, we also reduce the number of updates that are needed when recalibrating the photometry. We have put some examples of linking tile detections to pawprint detections in the VSA SQL cookbook ${ }^{11}$.

In Fig. 4 we show an entity-relationship model (ERM) for these new tables, showing how they are related to the current Multiframe, MultiframeDetector and Detection tables. The caption describes the relationships.

Tile and pawprint detections are matched within a fixed radius of $0.8^{\prime \prime}$ in VISTA, which is approximately the average seeing and is many times the typical astrometric error but less than the separation between easily-resolvable neighbouring objects, so objects will only be matched to the same object on different images, not to neighbouring objects. The matching algorithm is the same as for sources in the Source table, see Hambly et al. (2008). Like MergeLog, TileSet includes all the associated frames as a frame set consisting of the tile frame (tlmfID) and the 6 pawprint frames (o1mfID - o6mfID) where o1 is the pawprint with offsetID $=1$. TilePawPrints then contains the matched detections between the 7 frames, just like Source would have the matched detections between all the different filters. These tables should be used as linking tables to compare the tile detections to pawprint detections or even pawprint to pawprint detections between offsets.

There is an important difference in the way that tile sets are produced that causes some peculiarities to exist in the TilePawPrint tables that are not present in the SynopticSource tables. Tile sets are merged from frames of two different types - a tile and six pawprints - whereas synoptic framesets are always merged from one frametype, either pawprints in WFCAM or tiles in VISTA. In either case, each frame in the synoptic frame-set is similar to each other and the matching condition is a combination of multiframe identity and extension number. Non-detections in a particular filter have a default entry in the SynopticSource table: if there is no detection in the $J$-band then jSeqNum is simply set to the standard integer default value (-99999 999).

Since tile sets in TileSet are made up of a tile and six pawprints, with the tile composed of and overlapping all 16 detectors in each pawprint, tile sets cannot be matched by extension, but

\footnotetext{
11 http://surveys.roe.ac .uk/vsa/sqlcookbook.html\# TilePawprints
}

must be matched by multiframe alone. Therefore it is not possible to have entries in the tile set identified by multiframeID and extNum, they must be identified by multiframeID only, and the links in TilePawPrints must include both the extension number and sequence number. This makes the assignment of default rows more complicated. For example, if there is no detection in a particular frame, such as pawprint offset 1, we do not know offhand - without doing additional processing - which extension the detection should have been on (if any, since it may be in a gap between the detectors for this offset). We should set the default row for this missing detection as o1mfID = multiframeID of the pawprint, o1ExtNum = -9999, o1SeqNum = -99999 999 . However, there is no equivalent row in the Detection table because the foreign key constraint between the Detection table and MultiframeDetector forbids this, since there are not rows in MultiframeDetector with a non-default multiframeID, and a default extNum. Instead we set additional default rows in TilePawPrints, to have extension numbers equal to 2 and default sequence numbers, so that they can match with default rows in the Detection table. 2 is used because it is the lowest number of a real science extension, and can apply to both tiles and pawprints. These defaults are extremely useful in queries that compare the photometry of tile-detected sources in the merged-band catalogues to the pawprint detections. If a query compares the photometry of the tile to each of the 6 pawprints, in most cases one or more pawprints will not overlap with a particular tile detection; without these defaults no row would be returned even if all 5 other pawprints had a match and using these defaults, it is clear from the seqNum value that it is a non-detection. We must emphasize that an entry in TilePawPrints, which has a key $($ o1ExtNum $=2$, o1SeqNum $=-99$ 999 999) simply means that this object was not detected in pawprint offset 1 . It does not denote that that the object overlapped with extension 2 of pawprint offset 1: more than likely it was from part of the tile which did not overlap with pawprint offset 1 at all, although it could just be too faint to be detected in the pawprint.

Most TilePawPrints rows will be entries where the tile and two pawprints have matched detections, some where the tile and just one (in the outer strips) or three four, five or (infrequently) six pawprints have detections, some where the tile only has detections (usually at the faint end). Defaults are added as above where no detection exists. Careful selection of what is default and what is not will optimise the use of these tables. Any attributes in the detection tables can be compared in this way, although it is necessary to match a new instance of the Detection table for every frame in the table. Since this is done via the primary key (multiframeID, extNum, seqNum), the joined SQL queries are very efficient.

To match tile detections in a Source table or SynopticSource table to the detections on the constituent pawprints, it is necessary to remove the pawprint-only detections to leave a table with only the good tile detections and necessary defaults. If a query retains the pawprint-only detections, they are interpreted as defaults, so if a detection is missing in a particular filter it will be matched to every set of pawprint detections that are not linked to a tile-detection, a nonsensical result. To avoid this, we have created a view TilePawTDOnly that can be directly matched in the same way as TilePawPrints. However, for very large datasets, such as the VVV, queries work better if users use vvvTilePawPrints and add the necessary constraints into the where clause, see example queries found in the SQL cookbook. The ERM for the matching of the Source table to the pawprint detections is shown in Fig. 5. 


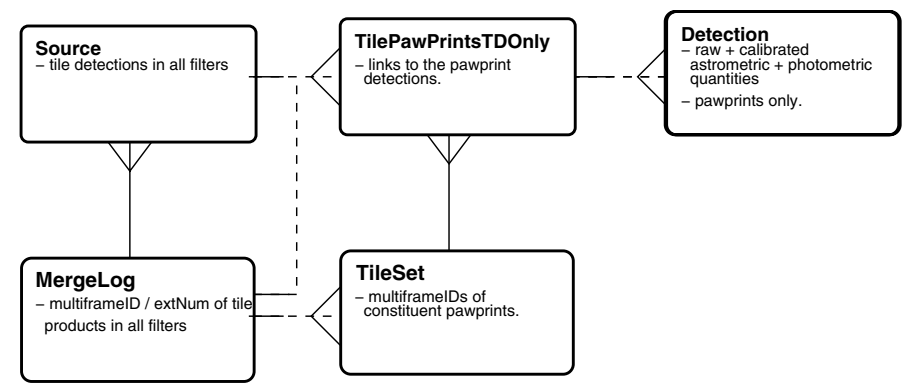

Fig. 5. ERM for the tile-pawprint linking tables matched with the Source table. Detections in the Source table come from tiles. In each MergeLog row there will be at least one non-default tile with a maximum equal to the number of filters used in the programme. Each entry in MergeLog, including defaults is matched to a TileSet and each Source row to a similar number of rows in TilePawPrintsTDOnly, some of which may be default depending on whether there was a detection in each observed filter. The relationship between MergeLog and TilePawPrintsTDOnly is necessary because the multiframe extension information in MergeLog is found in TilePawPrintTDonly, not TileSet.

In the case of the VHS, which has a single epoch only, we have provided an additional table vhsSourceXPawPrints which is a neighbour table between vhsSource and the pawprint detections in vhsDetection. This simply matches all pawprint detections within a given radius to a source. A typical query is shown at the bottom of the tile-pawprints section of the SQL Cookbook. It is more difficult to do precision queries on particular offsets or extensions as with TilePawPrints, but it is possible to do faster queries and may be preferable if only the pawprint data are required.

\section{Changes to image metadata}

\subsection{ESO attributes}

VISTA data pass through an ESO quality control pipeline (whose modules are provided by VDFS) in Garching before being ingested into the VDFS data processing pipeline in Cambridge, while the VDFS-generated data products supplied to the ESO SAF must comply with ESO metadata standards. As a consequence, the VISTA data products present contain a quantity of standard ESO information not present in WFCAM data products. For example, the headers of image files contain a number of ESO hierarchical FITS keywords ${ }^{12}$. Those required for data processing, or judged to be scientifically useful, are propagated into the Multiframe or MultiframeDetector tables for keywords from primary or extension headers, respectively, while the remainder are recorded in MultiframeEsoKeys and MultiframeDetectorEsoKeys tables.

Initial quality control occurs when the data is checked at the telescope and then at Garching to determine if the data was taken within the required constraints. This results in additional quality control metadata created for VISTA, which are included in the VSA, and which are not found in the WSA. These include OBSTATUS ("Completed", "Executed", "Aborted", "Pending" and "Undefined"), and ESOGRADE ("A", fully within constraints; "B", mostly - 90\% - within constraints; "C"; "D"; "R", rejected). If the OBSTATUS is not completed, the whole OB will be repeated later. Each OB is also quality assessed more generally when processed in Cambridge.

\footnotetext{
12 http://heasarc.gsfc.nasa.gov/fitsio/c/f_user/node28. html
}

There are also requirements for the files that are imported into the ESO Science Archive Facility ${ }^{13}$. We generate the following required keywords for the FITS files that are sent to the ESO archives: ABMAGLIM, ABMAGSAT, MJDEND, TEXPSUM, respectively representing the calculated $5 \sigma$ magnitude limit of the point sources in the extension in $A B$ mag, the $A B$ magnitude at which sources start to saturate, the end time (modified Julian days) of the last exposure which went into the stack and the summed total exposure of all the pawprints which went into the tile in seconds. These have been added into MultiframeDetector as abMagLim and abMagSat and Multi frame as mjdEnd, totalExpTimeSum. Currently the $A B$ saturated magnitude is only calculated when images are released to ESO, so all the values are default in the archive. The image pixel data have also been scaled and converted to 32-bit integer from 32-bit floating point, as a requirement for the ESO archive. The calculation of the scaling parameter is shown in Appendix A.

\subsection{Deprecations}

Deprecation codes in the Multiframe, MultiframeDetector, Detection tables are used to control which frames are used where. The different sub-surveys within UKIDSS followed the same deprecation policy, so it was possible to apply that uniformly across the whole WSA and not release any deprecated data. For VISTA, however, the Public Survey teams have defined different deprecation criteria, so it is not possible to define an analogous uniform policy for the VSA. Instead, it has been decided to release all data (deprecated or not) but to define additional deprecation codes to indicate whether or not particular images have been omitted in the creation of higher order products: deep stacks, tiles or mosaics, Source tables, SynopticSource tables, neighbour tables or multi-epoch/variability tables. The presence of most codes does imply exclusion from further use, but the following codes, which are exclusive to the VSA, are more nuanced:

- 50: the frame is good enough for single epoch measurements, but should not be used in a deep stack.

- 51: the frame has a problem with intermittency problem with channel 14 (some early frames had this temporary issue in detector 6). During deep stack creation, channel 14 is set to zero weight in a temporary confidence image.

- 53: these are frames where the quality is marginal. Do not use the frames in deep stacks, or use the detections in the variability statistics, but do link them in the best match table, so the the survey team can do more tests to determine whether they are good enough to be used in future releases.

The following codes, are standard exclusion codes, but are not found in the WSA:

- 55: aborted OB, if the science team decided they want to deprecate based on OBSTATUS.

- 56: deprecated because of poor ESOGRADE, if the science team decided they wanted to deprecate based on this.

- 58: deprecated because the catalogue could not be ingested. This happened for many very dense fields in early processing versions, but these have since been replaced. We have included the code in this paper for the sake of completeness and for users who use older team releases that still contain data annotated with this code. If a similar situation arises in the future, more data may be deprecated with this value.

${ }^{13}$ http://archive.eso.org/cms/ 


\section{Changes to catalogue attributes}

Some of the catalogue attributes present in the VSA differ from those in the WSA, for several reasons. Differences in the VISTA and WFCAM detector properties mean that different effects need to be flagged, while the observing strategy changes described in Sect. 2.1.1 led to requirements for different information. We have also introduced additional attributes in response to user demand and in the light of enhancements we have made, especially in the treatment of multi-epoch data.

We describe all these changes in the remainder of this section.

\subsection{VIRCAM detector properties}

The Raytheon VIRGO detectors used in VIRCAM have no detectable crosstalk and much lower persistence than the Rockwell Hawaii 2 detectors used in WFCAM. For this reason, detections do not have their quality bit-flags set for crosstalk (bit 19 of the post-processing error bit flags - ppErrBits, see Hambly et al. 2008) in the VSA, which reduces processing time. However, the VIRCAM detectors have a narrower dynamic range, with non-linearity and saturation occurring at lower flux levels. Nonlinearity is calibrated out as part of the VDFS pipeline at CASU, and WFAU have applied a saturation correction (Irwin 2009), where necessary, to the photometry in the VSA - note that users wishing to extract photometry for objects brighter than $m \sim 13$ (though this limit is survey dependent according to the DIT and filter used in the OBs) should use pawprint detections rather than tile detections since the corrections for saturation are more accurate for pawprints.

On the top half of detector 16, the quantum efficiency (QE) varies on short timescales making flat fields inaccurate. This is particularly noticeable at short wavelengths $(\sim 1 \mu \mathrm{m} \mathrm{e.g.} Z$ and $Y$ ). Since tiles are produced from 6 pawprints, each with 16 detectors, we still create a tile even if one or more of the constituent detectors has problems. Each tile pixel comes from up to 6 pawprints, which may have different PSFs (this is why tiles are "grouted" as described earlier). Tile detections that come partly from detector 16 in one or more pawprints are flagged using the post-processing error flag (ppErrBits) bit 12, so that users can select a data set without these detections or with them, whichever they prefer. Many of these detections have a low average confidence. We also added a flag for low confidence detections, bit 7 .

Occasionally a particular pawprint detector is deprecated for one of several reasons (Hambly et al. 2008), e.g. poor sky subtraction, bad channels, detector was not working correctly at the time of observation. The confidence of a deprecated detector is set to zero when making the tile, and this produces poorly defined extractor values (infinities and not-a-number), which are ingested as defaults into the database. These tile detections are flagged with bit 24 .

The two strips at the top and bottom of the tile have half the exposure time of most other parts of the tile. We flag these with bit 23. This is partly for the users and partly so that these detections do not become primary sources in the Source table of the survey if they overlap with a full exposure region of another tile.

The list below is a summary of the new detection quality bitflags developed for VISTA tile detections.

- Bit 7, Low average confidence $(<80)$ in default aperture.

- Bit 12, Source image comes partly from detector 16 .

- Bit 23, Source lies within a strip of the tile that has half the exposure of most of the tile.
- Bit 24, Source lies within an underexposed region due to a missing or deprecated detector.

These tile flags have not been applied to catalogues from external mosaics created for VIDEO, since their processing is quite different.

\subsection{Changes to attributes in Detection tables}

The VDFS extractor (Irwin et al. 2004), which generates the raw catalogue parameters for all the VSA data (apart from VIDEO mosaic catalogues, which are extracted using Source Extractor; Bertin \& Arnouts 1996) has had a few modifications so that there are slightly different output parameters for VISTA than WFCAM. In the original FITS catalogues produced by the VDFS extractor the Parent_or_child column (deblend in the WSA Detection tables) has been replaced by average_conf (stored as the avConf in the VSA Detection tables), while the Hall radius, Hall flux and Hall flux error have been replaced by a half-light radius (halfRad) and flux and flux error (halfFlux, halfFluxErr) within an aperture twice the half-light radius.

\subsubsection{Modified Julian Day}

In WFCAM, we used the mjdObs in Multiframe as the time for each observation, which was used in light-curves. This attribute is inadequate in VISTA though, since tiles are made from overlapping pawprints which each have different mean observation times and each tile detection may come from a different combination of pawprints. This is particularly important for surveys such as the VMC (Cioni et al. 2011), which require a significant fraction of an hour of integration time to reach the required depth at each epoch, but are looking for variables with periods of a few hours. In this case an accurate measurement of the mean observation time is fundamental to the science.

We have now added a new attribute mjd into the detection tables. This is the standard Modified Julian Date (MJD, in double precision days since midnight on Nov. 17th 1858) and is calculated detection by detection in the case of tiles or extension by extension for pawprints. During "grouting", the average MJD of each tile detection is calculated as the weighted average (weighted by the average confidence in a 1 " radius aperture: aperture 3) MJD of the different pawprints that contributed to the tile detection. In the FITS file, this is expressed as a 4-byte floating point value in minutes from the beginning of the day of the observation, as column MJDOff, with the beginning of the day given in the header as MJD_DAY. We have also calculated the mean MJD for each pawprint detector and added this to MultiframeDetector as mjdMean. This is the value that becomes the mjd (8-byte double precision) value in the detection tables for pawprints, not the mjdObs, which is the start time of the observation.

\subsubsection{Half-light radii}

As well as ingesting detection attributes calculated by the VDFS extractor produced in the FITS catalogue output, other attributes are calculated by the archive curation software. These include several half-light radius measurements, based on the aperture fluxes and the Petrosian flux, using the same method discussed in Smith et al. (2009). These new attributes are:

- hlCircRadAs, the half-light circular radius in arcseconds. 
- hlCircRadErrAs, the error in the half-light circular radius in arcseconds.

- hlGeoRadAs, the geometric mean between the half-light radius along the semi-major axis and the half-light radius along the semi-minor axis in arcseconds.

- hISMnRadAs, the half-light radius along the semi-minor axis in arcseconds.

- hlSMjRadAs, the half-light radius along the semi-major axis in arcseconds.

- hlCorSMnRadAs, the half-light radius along the semiminor axis corrected for seeing, in arcseconds.

- hlCorSMjRadAs, the half-light radius along the semi-major axis corrected for seeing, in arcseconds.

The algorithms used to calculate the above attributes are given in Appendix B.

\subsubsection{Magnitude corrections}

Photometric calibrations, derived by the VDFS pipeline at CASU (Hodgkin et al. 2012, in prep. ${ }^{14}$ ), are applied in the archive curation software. As mentioned in Sect. 6.1, we now include a saturation correction to the pipeline produced magnitudes of stars flagged as potentially saturated. We decided to include explicit columns that contain this and other source dependent corrections (those that are not simply field dependent), making it easy for users to understand and apply the corrections themselves.

The current corrections that are applied to the magnitudes by WFAU in the VSA are:

- illumCorr, the illumination or scattered light correction that is calculated and provided by CASU for fields on a month by month basis.

- distortCorr, the radial distortion correction, which depends on the distance from the optical axis and the filter only.

- saturatCorr, the saturation correction, that is added to the 1 arcsec radius aperture magnitude (aperMag3) of bright stars only (those that are flagged as potentially saturated).

- deltaMag, the sum of the exposure time correction (2.5 $\log _{10}$ expTime), the atmospheric extinction correction $((0.5$ (amStart + amEnd $)-1)$ extinctionCat $)$, the illumination correction and the radial distortion correction. The saturation correction is not included, because it only applies to aperMag3. A user can calculate their own magnitudes on the VISTA photometric system for objects by measuring a flux in any way they like and applying the zero-point and adding deltaMag.

The aperture corrections are not included since they are only applied to specific magnitudes and are the same for all objects on one detector. The values for these are included in the MultiframeDetector table.

WFAU had several requests for aperture magnitudes without the point-source aperture correction (i.e. for extended sources). Therefore we have included these values for the 7 apertures (1-7) for which aperture corrections have been applied as standard. These are named aperMagNoAperCorr1, aperMagNoAperCorr2 to aperMagNoAperCorr7. These magnitudes can be used for extended sources if required.

\footnotetext{
${ }^{14}$ http://http://casu.ast.cam.ac.uk/surveys-projects/ vista/technical/photometric-properties
}

\subsection{Changes to attributes in the Source tables}

Several of the attributes in the Detection table have been propagated through to the Source table or SynopticSource table. Of the new Detection attributes, we have propagated hlCorSMjRadAs and the non-aperture corrected aperture magnitudes into the Source table. We do not propagate mjd though, since the sources in the Source table come from the deepest data, stacked across multiple epochs where the time of observation is not particularly useful. Since the SynopticSource matches data with a specific epoch, and is particularly useful for variability work on point sources, mjd is propagated but hICorSMjRadAs and aperMagNoAperCorr[1-7] are not.

We still only produce one Source table, from the highest (most processed image, i.e. tile if the survey contains both tiles and stacks, mosaics if the survey contains these) product layer (see Sect. 7). Producing one for tiles and one for pawprints breaks the idea of a single master source list (see Cross et al. 2009). Instead, we have a master source list produced from tiles which is linked to pawprints using the TilePawPrints table, see Sect. 4.1.1.

\section{Infrastructure}

There have been several changes to the science archive infrastructure that improve curation of the surveys, but can also be useful for scientists who want to make the best use the VSA. Some of these changes have been incremental and have been documented in Collins et al. (2009); Cross et al. (2009, 2011). The main changes to the VISTA Public Surveys from the UKIDSS Public Surveys are listed below:

- Automatically set up all the requirements, the database schema and curation tables contents using available data and basic programme properties from the Programme table. This was also done for the UKIDSS-DXS and WFCAM PI programmes.

- Manage multiple layers of products: pawprints, tiles and mosaics, including external products automatically.

- Have a more sophisticated setup for multi-epoch products, specified by the synopticSetup string in Programme.

We set up all the requirements, the database schema and curation tables contents for a survey, when we start preparing a static release (e.g. VHS-DR1), using a combination of the programme requirements in the Programme table and the available data. We have made the infrastructure and processing the same for all surveys, unlike in UKIDSS where the wide shallow surveys (GPS, GCS and LAS) were processed differently from the deep surveys (DXS and UDS). This makes it easier for the operators who run tasks, and makes it much simpler if programmes evolve in the future. For instance, if the VHS decided to add in a second epoch in any filter, this would be automatically accommodated.

\subsection{Stack, tiles and mosaics}

Requirements for stack, tile and mosaic products are set up by grouping the data into different pointings by position, position angle and, in the case of pawprints, offset. The requirements for a particular release are stored in RequiredStack, RequiredTile, and RequiredMosaic. The stacking software uses the definitions to create the deepest stack possible for each product in RequiredStack from the pawprints. The tiling software creates tiles at each location in RequiredTile using these 
stacks, so the tile requirements must be linked to the pawprint requirements.

The different layers of products (pawprints, tiles and mosaics) can be linked to each other using the ProductLinks table: e.g. tile productID 1 in RequiredTile in the VHS may be composed of pawprints with productIDs 1, 3, 5, 7, 9 and 11 in RequiredStack. ProductLinks links the requirements whereas Provenance links the image metadata from the actual files. From one release to another the values in ProductLinks, RequiredTile and RequiredStack may stay the same (although this is not guaranteed), but a product which initially contained 2 epochs worth of image data may be replaced by one containing 5 epochs worth of image data and will therefore link to different multiframes in Multi frame and Provenance.

External (made outside VDFS) products, e.g. VIDEO (or UKIDSS-UDS in the WSA) mosaics, which are created by the survey team and imported into the VSA, are set up via the ExternalProducts table, which contains the programme, product type, release number and information about who created the mosaic.

The required products and the actual image frames are now linked to each other via the ProgrammeFrame table which includes programmeID, productID and releaseNum and links to the image metadata tables via multiframeID. The release number for products is a running number from when WFAU first started producing releases for the science teams, so the products in the first public releases of the VISTA Public Surveys have a variety of release numbers depending on the programme. In VISTA, the programme translation for each incoming FITS image is more complicated than for WFCAM, and the programme matching algorithm uses a combination of the HIERARCH ESO OBS PROG ID, HIERARCH ESO OBS NAME and HIERARCH ESO DPR CATG header keywords.

ProgrammeFrame is essential for keeping track of what images are related to each requirement. This makes it much easier for scientists and VSA support staff to keep track of what has been created and whether anything is missing. This infrastructure is crucial for the automated curation (Collins et al. 2009) of VSA products, where decisions are made about what tasks need to be run based on the requirements and what has already been completed.

When OB frames are recalibrated in multi-epoch programmes, the OB tiles are compared to the deep tiles and the zero-points adjusted accordingly. A change to the zero-point of the tile is propagated to the constituent pawprints. The code to propagate the zero-point differences was not developed until very recently, so most datasets in the first release will not include this propagation; at the time of writing only the VVV dataset will include this. The pawprint zero-points for the other multi-epoch public surveys (VIKING, VMC and VIDEO) will be correctly updated for all recalibrations in the data releases that contain data from ESO semester P87 and beyond.

\subsection{Multi-epoch tables}

We have introduced a new string attribute into the Programme table, called synopticSetup, to control the production of more than one Best Match table, i.e. both a SourceXDetectionBestMatch and a SourceXSynoptic SourceBestMatch. In surveys such as the VVV, many scientists would like colour information for variable stars, so the colours must come from near-contemporaneous observations. This information is in the SynopticSource table whereas the colours in the Source table come from the deepest images which are stacked from several epochs of data and are certainly not contemporaneous. However, this survey will take many tens of epochs, mostly in one filter, $K_{\mathrm{s}}$, so a SynopticSource table that covers the full time range would be inefficient - the $Z, Y$, $J$ and $H$ band attribute columns would contain mainly default values. A more efficient way is to specify the SynopticSource over a short time range and specify that the statistics in the Variability should come from data in SourceXDetection BestMatch, which covers the whole time range. It is still necessary to link the SynopticSource table with all the other tables, so a SourceXSynopticSourceBestMatch table is required too.The synopticSetup attribute is a string, with the following value in the VVV: BOTH: VAR-UNC:COR, SV,P87, which can be parsed to give the following information: create both Best Match tables; use the uncorrelated (SourceXDetectionBestMatch) when calculating the variability statistics, and only use data between the beginning of the Science Verification period (SV) and the end of ESO Period 87 (P87) in the correlated table (SourceXSynopticSourceBestMatch).

\section{Other recent improvements to the VSA and WSA}

In addition to the above changes necessary for processing VISTA data, we have made various changes to improve overall curation of WFAU products. These extend the database design described in Hambly et al. (2008) and Cross et al. (2009).

\subsection{Improvements to multi-epoch data model and calculations}

\subsubsection{Creating the BestMatch tables}

In Cross et al. (2009, Sect. 9.2), we discussed possible improvements to checking missing observations to correctly create the BestMatch tables. One method that we discussed possibly implementing was the half-space method (Budavári et al. 2010), which we have now implemented. We define 16 half-spaces for each single epoch image. These 16 half-spaces come in 4 sets, one 2 pixels outside each image edge, one 2 pixels inside each image edge, one 2 pixels outside the edge of the jittered region where the exposure time per pixel goes from the total exposure time to some fraction of it and one 2 pixels inside this edge. With 4 edges, this gives 16 half-spaces. We found that defining a halfspace using 3 points: the two ends of an edge and the midpoint, the edge could usually be described to an accuracy of around one pixel, so a 2 pixel margin each side would encompass all points which we were unsure about. Each half-space is described using 4 numbers, a 3D Cartesian vector, normal to the plane of the half-space and a constant that gives the offset of the plane from the centre of the sphere. All the half-space information is stored in a new table, AstrometricInfo (one for each multi-epoch programme, e.g. vmcAstrometricInfo), to help with the curation of multi-epoch data. As well as the half-space information, we have included place-holder columns for attributes to describe small adjustments to the astrometric solution of each image that will improve fitting to the proper motion, when we start calculating proper motions in VISTA data (see Collins \& Hambly 2012, for the description of the method used for wide area UKIDSS surveys).

The half-spaces are used to check frames which do not have an expected match to a primary source in the Source table, (see Cross et al. 2009, Sect. 9.2). If there is no detection, this may be for one of several reasons: the frame does not overlap with 


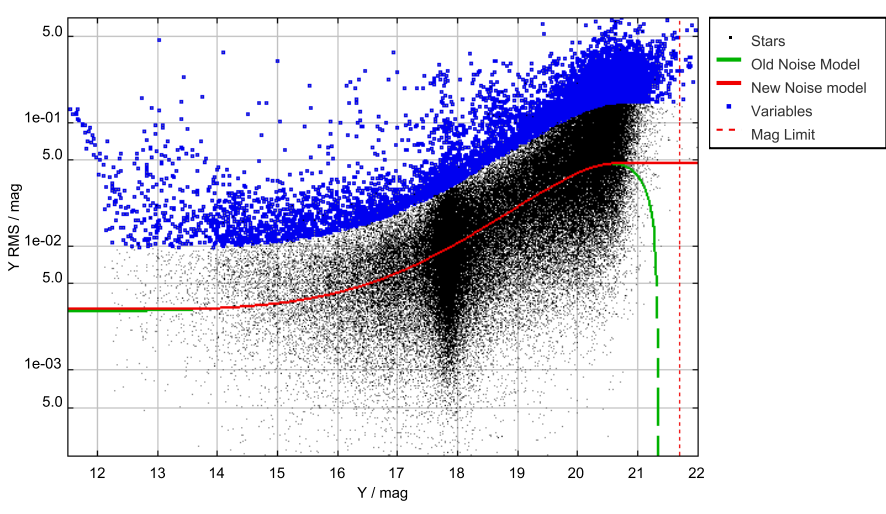

Fig. 6. Magnitude-rms plot of a dense pointing in the VMC, where the fitting function turns over before the expected magnitude limit (dotted vertical line). The old expected rms as a function of magnitude is plotted as the dashed line, and the new expected rms as a function of magnitude is plotted as the solid line. Outside of the fitting range, or beyond the turnover point, the rms is a constant. Non-variable stars are shown as dots, and variables are shown as open squares. At the bright end, $Y<$ $12.5 \mathrm{mag}$, the rms increases due to saturation effects, but this has not been included in the model yet.

the part of the sky containing the source; the source is within the jitter regions, where the integration time is less and there may be a gradient in the integration time across the object; the source is too faint to be detected on a single exposure; the source is usually bright enough, but has faded below the detection threshold; the source is blended with another; the object has moved sufficiently far from the expected position.

Using the half-spaces allows us to flag the first two possibilities. Checking whether a detection should be within the image or within the jitter section is trivial and each calculation is extremely quick. Most importantly, since the half-space describes an edge accurately within a pixel or two, very few objects need the more careful test that use the WCS to calculate the exact position of the object on the frame. Using the half-spaces we are able to reduce the number of slow tests to only those objects within two thin strips, each 4 pixels wide, at the image edge and the edge of the jitter region. The half-space information is stored so that archive users can use it too, to rapidly determine whether an object is within the frame.

\subsubsection{Expected noise model}

We have made changes to the calculation of the expected noise. The expected noise is still based on fitting a function, in this case, a Strateva function to the rms versus mean magnitude data (see Cross et al. 2009). In early team data releases (before version 1.1 data was released), the expected noise was simply the value of this function at the mean magnitude of the source. However, we found that the actual magnitude limit was often quite a bit brighter than the expected magnitude limit, especially when the field is very dense, see Fig. 6. When this happens the rms versus magnitude plot turns over and simply using the fit will underestimate the rms; indeed sometimes the expected rms will be negative. To mitigate against this, we have made the following changes:

- Calculate the turnover point: the maximum rms as a function of magnitude if the function does turn over.

- For all magnitudes fainter than the turnover point or the maximum range of magnitudes in the fit (whichever is brightest), set the expected rms to the value at this point, see Fig. 6 .
- For all magnitudes brighter than the minimum range of magnitudes in the fit, set the expected $\mathrm{rms}$ to the value at this point.

The astrometric fit has also been updated. Instead of calculating a simple clipped mean, we calculate a weighted mean position. We calculate the expected astrometric noise in each filter, in the same way as we calculate the expected photometric noise, using the angular separation between each epoch position and the median absolute deviation clipped median position for stationary stars and fitting a function to these median values as a function of magnitude. This function describes the locus of the astrometric uncertainty for non-moving point sources, much as the equivalent fit for the magnitude rms described the photometric uncertainty as a function of magnitude for non-variable point sources. This calculated uncertainty as a function of magnitude will be used to weight the position.

A particular pointing may only be observed once in one filter, and in some programmes a filter is only observed once in each pointing, so it is often not possible to fit a noise model for each pointing and filter. For photometric variability statistics, this is not a problem, since all the values are default if there is only one epoch, but when it comes to the astrometric fit, we would like to use all the data in all filters together, to improve the fit.

To estimate the errors on these frames, we calculate a default noise model in each filter which has a calculable noise in at least one pointing. We take all the calculated noise models, and calculate the mean rms of these models in each of a set of nine bins across the magnitude range, and fit the noise model to the mean rmss. This model is used in any pointing in this filter where there is only one epoch.

For filters where there is only ever one epoch, we cannot directly measure the noise as a function of magnitude, so we make the assumption that the behaviour in any filter is similar to the others (which is born out by experience of surveys where multiple observations are taken in all filters). We expect that the limit reached at the bright end for the same DIT in the OBs will be the same and that the increase in noise towards the faint end depends on the depths of the exposure, which depends on the total exposure time or expected magnitude limit. Moreover, any other differences, such as the effects of sky brightness or residual non-linearity are likely to be a function of wavelength, so we choose the nearest filter in wavelength that has enough epochs for a fit to be made to the rms as a function of magnitude. We take the default model in this filter and adjust for the difference in expected magnitude limit, e.g. using the Strateva model, we will calculate new values for $b$ and $c$ as follows

$$
\begin{aligned}
\langle\zeta(m)\rangle & =a+b 10^{0.4 m}+c 10^{0.8 m} \\
\Delta m & =m_{1}-m_{1}^{\prime} \\
a & =a^{\prime} \\
b & =b^{\prime} 10^{-0.4 \Delta m} \\
c & =c^{\prime} 10^{-0.8 \Delta m}
\end{aligned}
$$

where $m_{1}$ is the magnitude limit of frames in this filter and $m_{1}^{\prime}$ is the magnitude limit of frames in the comparison filter.

The weighted mean position uses a $3 \sigma$ clipped weighted mean in each of the three Cartesian coordinates, and then converts back to equatorial coordinates. The type of fit used is recorded in the VarFrameSetInfo table as motionModel. The model described above is a static weighted model: "wgtstatic". When we have VISTA data over several years we expect to fit for proper motion too. 


\subsection{Improvements to the interface}

The VSA proprietary and public release databases can be accessed and queried via the web-browser based interface. The various access methods allow users to perform SQL queries on the science ready tables; extract image cut-outs and download entire image and catalogue files. In addition public releases will be accessible under the Virtual Observatory (VO). Releases will be discoverable in the VO registries. A table access protocol ${ }^{15}$ (TAP) interface to each data release will allow users to perform SQL queries using the astronomical data query language (ADQL). We already have partially compliant TAP services available for all our main data holdings. They can be accessed through software like TOPCAT (Taylor 2005) and VOExplorer, as well as through standard HTTP GET and POST commands. The services and their endpoints are registered on the major VO registries. We also have conesearch and simple image access protocol $\left(\mathrm{SIAP}^{16}\right)$ services available on the VO for the same list of datasets.

Fully compliant TAP services will likely be available by the end of 2012.

\section{Illustrative science examples}

\subsection{Colours of VHS point sources}

The optical-infrared colour-colour plot is a powerful classifier of different types of stars, with most stars lying along a narrow locus. However, extinction and poor photometry can widen this locus and prevent the separation of brown dwarfs, QSOs and compact galaxies. The following selection will select point sources in the VHS, which are matched to point sources in the SDSS and are not flagged for poor quality. We select the colours and positions, but only for stars in areas of low Galactic extinction.

SELECT S.SourCeID, S.RA, S.Dec,

$/$ * select position colour and magnitude information */

(sdPho.psfMag_g-s.jAperMag3) AS gmjPnt, jmksPnt, ksAperMag3

/* from vhsSource, SDSS DR7 PhotoObjAll, neighbour table */

FROM vhsSource as $s$,

vhsSourceXDR7PhotoObjAll as $\mathrm{x}$,

BESTDR7.. PhotoObjAll as sdPho

/* join the tables */

WHERE $s$. sourceID $=x \cdot$ masterObjID AND

x. slaveObjID=sdPho.objID

$/ *$ find matches within 2 arcsec */

AND $\mathrm{x}$.distanceMins $<=0.0333$

$/ *$ that are nearest matches */

AND $\mathrm{x}$.distanceMins IN (

* sub query to find minimum distance

for a match to this sourceID */

SELECT MIN(distanceMins)

FROM vhsSourceXDR7PhotoObjAll

WHERE masterObjID=x.masterObjID)

/* select SDSS primary objects and stars */

AND x.sdssPrimary $=1$ and $x . s d s s$ Type $=6$

$/ *$ objects with no flags in VHS */

AND jppErrBits $=0$ AND ksppErrBits $=0$

$/ *$ stars or probable stars in VHS */

\footnotetext{
15 http://www . ivoa.net/Documents/TAP/

16 http://wWw . ivoa.net/Documents/SIA/
}

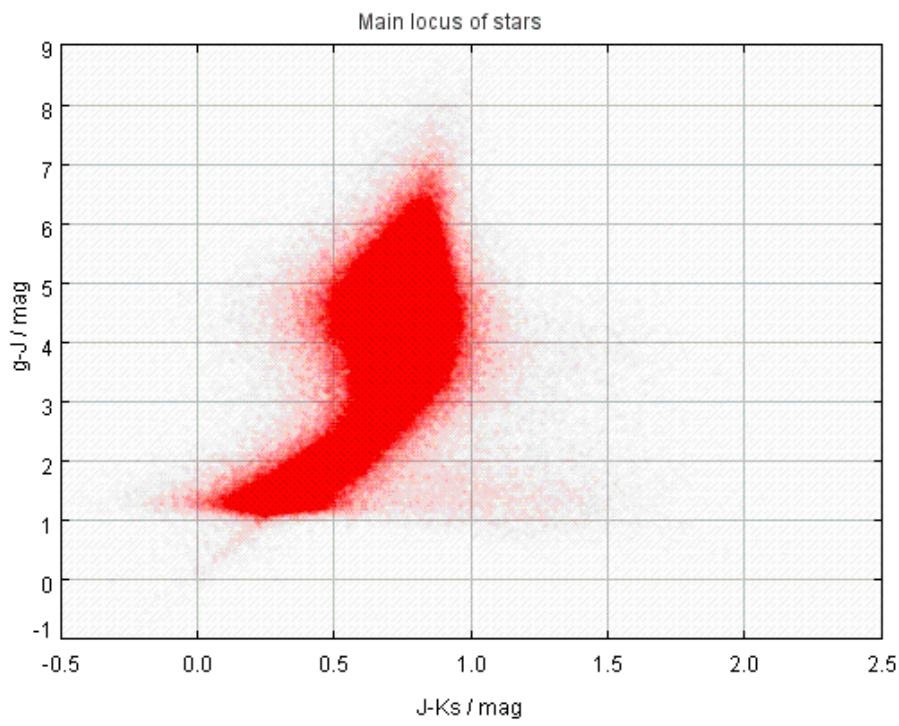

Fig. 7. Main locus of stars matched between the VHS and SDSS selected in $(g-J)$ versus $(J-K \mathrm{~s})$.

AND mergedClass IN $(-1,-2)$

$/ *$ Not default magnitudes in SDSS or VHS */ AND sdPho.psfMag_g $>0$. AND s.jAperMag $3>0$.

AND s.ksAperMag $3>0$.

These data can be plotted using TOPCAT (Taylor 2005), and the main locus can be found, as seen in Fig. 7. The gradient of the locus in the colour-colour plot can be measured and then rare objects can be further selected.

\subsection{Flare stars}

The following query selects objects that could be flaring stars or some type of cataclysmic variable. To do this, we select sources that have a minimum $K_{\mathrm{s}}$ magnitude that is at least 2 mag brighter than the median magnitude and further, it has at least 2 measurements that are brighter than the median by $0.5 \mathrm{mag}$. This second constraint should remove sources with one point that has escaped flagging. We also want at least $5 \operatorname{good} K_{\mathrm{s}}$ detections for a reasonable light curve. The following query was performed on the VVV survey.

SELECT v.sourceID, S.RA, S.Dec,

/* select some useful attributes, pointing info, number of observations, min, medium, maximum, variable class, and star/galaxy class */

v.framesetID, ksnGoodObs, ksMinMag, ksMedianMag, ksMaxMag, variableClass, mergedClass, (ksMedianMag-ksMinMag) as ksFlareMag, COUNT (*) AS nBrightDetections

/* from vvvVariability and vvvSource */ FROM vvvVariability as v, vvvSource as $\mathrm{s}$, vvvSourceXDetectionBestMatch as b, vvvDetection as $\mathrm{d}$

/* first join the tables */

WHERE $\mathrm{s}$. sourceID=v. sourceID AND b. sourceID= v. sourceID AND b.multiframeID=d.multiframeID AND b.extNum=d.extNum AND b. seqNum= d. seqNum AND

/* select the magnitude range, brighter than $\mathrm{Ks}=17$ and not default. */

ksmedianMag $<18$. and ksmedianMag $>0$. AND 


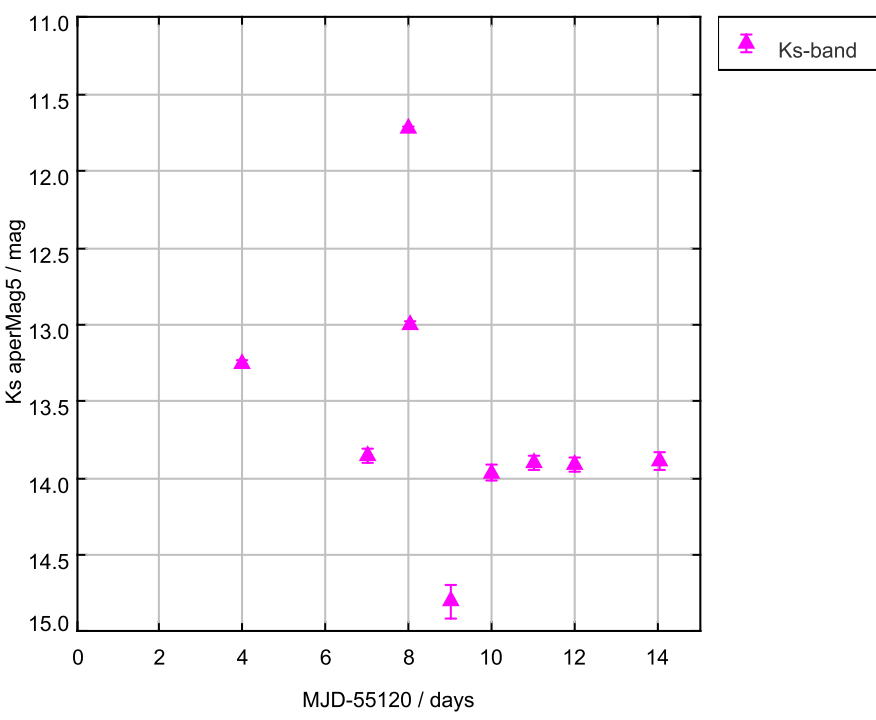

Fig. 8. Light curve of a flare star in the VVV selected using the query in Sect. 9.2.

\author{
/* at least 5 observations */ \\ ksnGoodObs $>=5$ AND ksbestAper $=5$ AND \\ /* Min mag is at least 2 magnitudes brighter \\ than median mag(but minMag is not default) */ \\ (ksmedianMag-ksminMag) $>2$. and ksMinMag $>0$. AND \\ $/ *$ Only good Ks band detections in same \\ aperture as statistics are calculated in*/ \\ d. seqNum $>\theta$ AND d.ppErrBits IN $(\theta, 16)$ AND \\ d.filterID=5 AND d.aperMag5 $>0$ AND \\ d. aperMag $5<($ ksMedianMag-0.5) \\ /* Group detections */ \\ GROUP BY v.sourceID, s.ra, s.dec, \\ v.framesetID, ksnGoodObs, ksMinMag, ksMedianMag, \\ ksMaxMag, variableClass, mergedClass \\ HAVING COUNT $(*)>2$ \\ $/ *$ Order by largest change in magnitude first.*/ \\ ORDER BY ksMedianMag-ksMinMag DESC
}

We plot the Ks-band light curve of one of these objects in Fig. 8. The majority of the detections are 14th magnitude, but there is a flare of almost $2.5 \mathrm{mag}$ followed by fading of $1 \mathrm{mag}$ before the star returns to $K_{\mathrm{s}}=13.9 \mathrm{mag}$.

\subsection{Global properties of VIKING-SDSS galaxies}

The following selection uses IR photometry and sizes from VIKING combined with optical colours and redshifts - both spectroscopic and photometric - from SDSS. In this query we use neighbour tables to join VIKING and SDSS. We use the SQL command "UNION" to combine the query which matches to galaxies with spectra to the query for those with only photometric redshifts. Users who are worried about completeness can use the "UNION" command to combine further queries, such as those that select for VIKING galaxies without SDSS matches or ones for SDSS matches but neither spectroscopic or photometric redshifts. The two combined queries must have the same number of columns, with the same names. In the cases where one query has columns which the other has no entry for (e.g. redshift, $z$ ) we can fill this column with a default number, just as we demonstrate below with the redshift status and spectroscopic type.

\section{SELECT}

$/ *$ select information necessary to create bi-variate brightness distribution, extinction corrected Petrosian magnitudes put into $\mathrm{AB}$ system and seeing corrected, semi-major axis size, PLUS SDSS colours and redshifts (spectroscopic and photometric)

$* /$

S. sourceID, s.RA, s.Dec, s. frameSetID,

(s.hPetroMag-s.aH+fh.VegaToAB) AS hPetroAB, s.hHlCorSMjRadAs, (s.hPetroMag+

$2.5 * \log 10(2 . * 3.14159 *$ s.hHlCorSMjRadAs*

s.hHlCorSMjRadAs) -s.aH+fh.VegaToAB) AS

hSurfBright, (s.ksPetroMag-s.aKs+

fks.VegaToAB) as ksPetroAB,

s. ksHlCorSMjRadAs, (s. ksPetroMag+

$2.5 * \log 10(2 . * 3.14159 *$ s. ksHlCorSMjRadAs*

s.ksHlCorSMjRadAs)-s.aKs+fks.VegaToAB)

AS ksSurfBright, dr7spec.objID as sdssID, ( (dr7spec.modelMag_u-dr7spec.extinction_u)(dr7spec.modelMag_g-dr7spec.extinction_g)) as umgModel, z, zErr, zConf, zStatus, specClass $/$ from vikingSource, Filter (one for each filter for VegaToAB), SDSS-DR7 neighbour table, SDSS SpecPhoto table */

FROM vikingSource AS s, Filter AS fh, Filter AS fks, vikingSourceXDR7Photo0bjAll AS xdr7, BESTDR7.. SpecPhotoAll as dr7spec

/* First join tables, */ WHERE xdr7.masterObjID=s.sourceID AND

fh.filterID=4 AND fks.filterID=5 AND

dr7spec.objID=xdr7.slave0bjID AND

$/$ * select VIKING primary sources matched to SDSS primary sources */

(priOrSec $=0$ OR priOrSec $=$ frameSetID) AND

sdssPrimary=1 AND

dr7spec.sciencePrimary=1 AND

$/ *$ within 2" of nearest match */

$\mathrm{xdr7}$. distanceMins $<0.03333$ AND

xdr7.distanceMins IN (

SELECT MIN (distanceMins)

FROM vikingSourceXDR7PhotoObjAll

WHERE masterObjID=xdr7. masterObjID AND sdssPrimary=1) AND

/* for objects classified as galaxies or probable galaxies in VIKING */

mergedClass IN $(1,-3)$ AND

$/ * \mathrm{~h}$ and ks size is $0.7<$ sma $<=10$. arcsec */ ksHlCorSMjRadAs $>0.7$ AND hHlCorSMjRadAs $>0.7$ AND ksHlCorSMjRadAs $<=10$. 0 AND

hHlCorSMjRadAs $<=10$. 0 AND

$/ *$ good quality data in VIKING $\mathrm{h}$ and $\mathrm{ks} * /$ hppErrBits=0 AND ksppErrBits=0 AND

$/ *$ ks extinction corrected $\mathrm{AB}$ mag $<20.5 * /$

(ksPetroMag-aKs+fks. VegaToAB) $<20.5$ AND

$/ * \mathrm{RA}$ and Dec range to restrict to where SDSS is */

S. RA $>100$. AND s. RA $<250$. AND s.Dec $>-5$. AND

I*z>=0.002*/

dr7spec . $z>=0.002$

/* Add in ones which do not have SDSS spectra using UNION */

UNION 


\section{SELECT}

/* select information necessary to create bi-variate brightness distribution, extinction corrected

Petrosian magnitudes put into $\mathrm{AB}$ system and seeing corrected, semi-major axis size AND SDSS matches to PhotoObj table and photoz table */ s. sourceID, s.RA, s. Dec, s. frameSetID,

(s.hPetroMag-s.aH+fh.VegaToAB) AS hPetroAB, s.hHlCorSMjRadAs, (s.hPetroMag+

$2.5 * \log 10(2 . * 3.14159 *$ S. hHlCorSM jRadAs* s.hHlCorSMjRadAs)-s.aH+fh.VegaToAB) AS hSurfBright, (s.ksPetroMag-s.aKs+fks.VegaToAB) as ksPetroAB, s.ksHlCorSMjRadAs, (s.ksPetroMag+ $2.5 * \log 10(2 . * 3.14159 * \mathrm{~s} . \mathrm{ksHlCorSMjRadAs} *$ s.ksHlCorSMjRadAs) -s.aKs+fks.VegaToAB) AS ksSurfBright, dr7phot.objID as sdssID, ( (dr7phot.modelMag_u-dr7phot.extinction_u)(dr7phot.modelMag_g-dr7phot.extinction_g)) as umgModel, photz.z as $z$, photz.zErr as zErr, -9.9999 as zConf, -9 as zStatus, -9 as specClass $/$ * from vikingSource, Filter (one for each filter for VegaToAB), SDSS-DR7 neighbour table, */

FROM vikingSource AS s,Filter AS fh,Filter AS fks, vikingSourceXDR7Photo0bjAll AS xdr7, BESTDR7..PhotoObjAll as dr7phot, BESTDR7...photoz as photz /* First join tables, */ WHERE xdr7.masterObjID=s. sourceID AND fh.filterID=4 AND fks.filterID=5 AND dr7phot.objID=xdr7. slave0bjID AND photz.objID= dr7phot.objID AND dr7phot.objID NOT IN (

SELECT dr7spec.objID

FROM BESTDR7.. SpecPhotoAll as dr7spec WHERE dr7spec.objID=xdr7.slave0bjID AND dr7spec.sciencePrimary=1) AND

/* select VIKING primary sources matched to SDSS primary sources */

(priOrSec=0 OR priOrSec $=$ frameSetID) AND sdssPrimary $=1$ AND

/* within 2" of nearest match */

xdr7. distanceMins $<0.03333$ AND

xdr7.distanceMins IN (

SELECT MIN(distanceMins)

FROM vikingSourceXDR7PhotoObjAll

WHERE masterObjID $=x d r 7$. masterObjID AND sdssPrimary=1) AND

$/ *$ for objects classified as galaxies or probable galaxies in VIKING */ mergedClass IN $(1,-3)$ AND $/ * \mathrm{~h}$ and ks size is $0.7<\operatorname{sma}<=10$. arcsec */ ksHlCorSMjRadAs $>0.7$ AND hHlCorSMjRadAs $>0.7$ AND ksHlCorSMjRadAs $<=10.0$ AND hHlCorSMjRadAs $<=10.0$ $/ *$ good quality data in VIKING $\mathrm{h}$ and ks */ AND hppErrBits $=0$ AND ksppErrBits $=0$ AND $/ *$ ks extinction corrected $\mathrm{AB}$ mag $<20.5 * /$ (ksPetroMag-aKs+fks.VegaToAB) $<20.5$ AND $/ *$ RA and Dec range to restrict to SDSS */ S. RA $>100$. AND s.RA $<250$. AND $s$. Dec $>-5$. AND $/ * z>=0.002 * /$

photz. $z>=0.002$

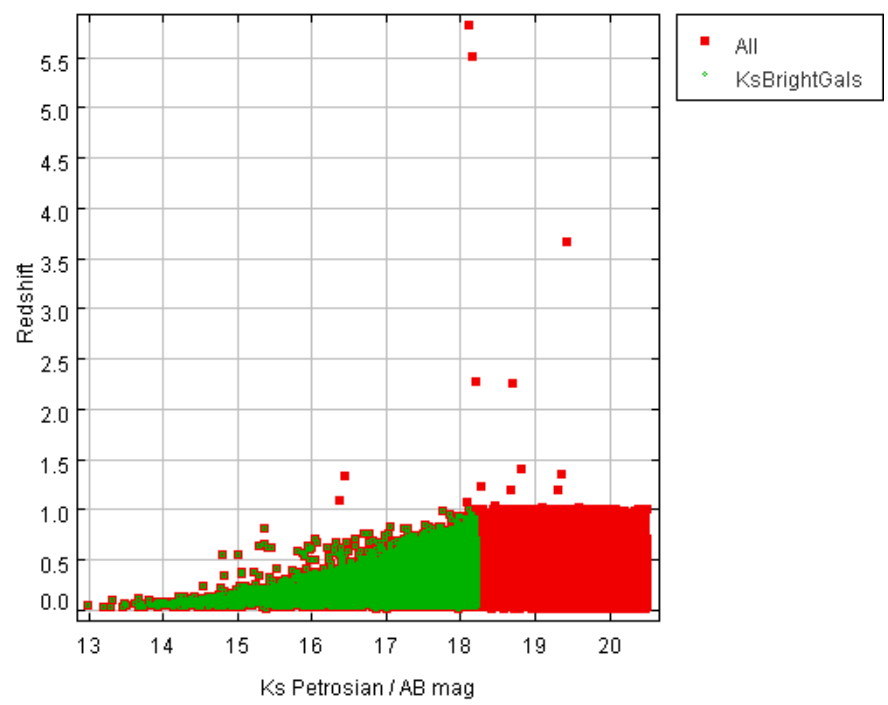

Fig. 9. $K$ s-band $A B$ Petrosian magnitude versus redshift. This plot shows very few redshifts greater than $z=1$, shown in red squares. The galaxies with $z>1$ are likely to have spectroscopic redshifts, whereas the photometric redshifts are limited to $0<z<1$. Galaxies with $K \mathrm{~s}_{A B}<18.2$ tend to have $z<1$, so we have selected a sample in TOPCAT which have $K \mathrm{~s}<18.2$ and $z<1$, which are shown in light green dots.

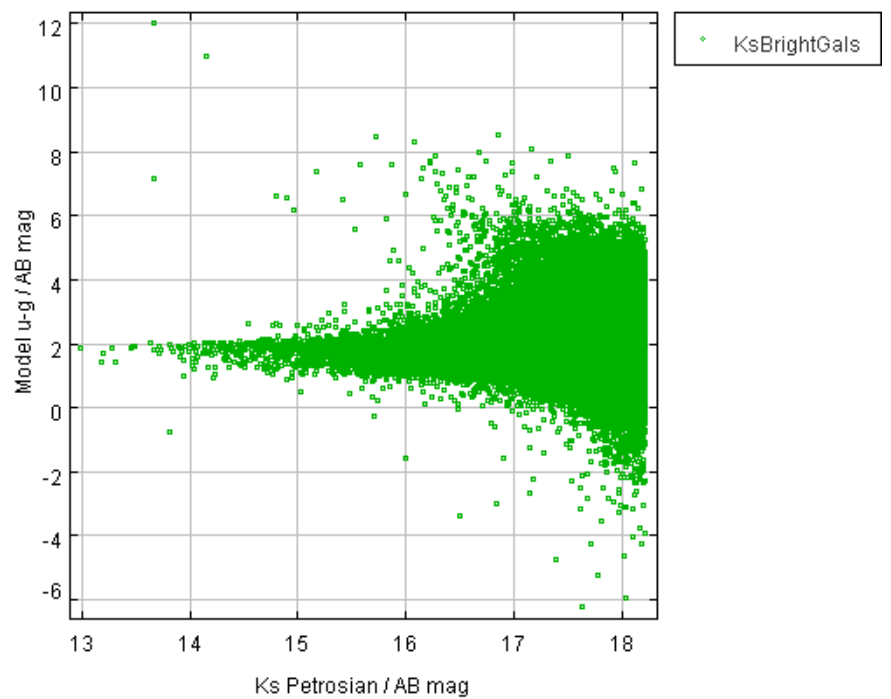

Fig. 10. $K$ s-band $A B$ Petrosian magnitude versus u-g model mag colour for the $K \mathrm{~s}_{A B}<18.2, z<1$ sample selected in Fig. 9. At the bright end, the red-sequence is clear, but at fainter magnitudes, galaxies will be at higher redshift, so the observed colours are less meaningful.

We plot the magnitude against the redshift for these galaxies, Fig. 9, and find that there is an artificial selection at $z<1$. The most likely explanation is that the photometric redshifts are limited to this range, since the SDSS optical colours do not give reliable photometric redshifts outside this range. By selecting a subsample at $K_{\mathrm{s}}<18.2$ and $z<1$, we have a more complete sample with reliable redshifts. We use this sample to look at the colour-magnitude plot and the surface brightness magnitude plot of galaxies, Figs. 10 and 11. The surface brightness, colour, magnitude and redshift are all fundamental for classifying galaxies. 


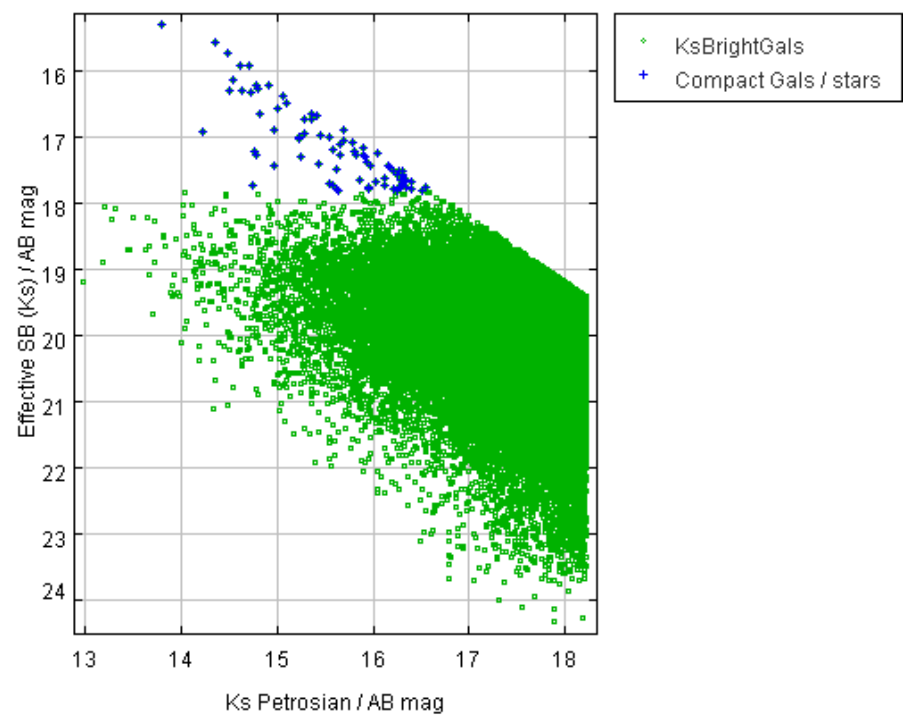

Fig. 11. $K$ s-band $A B$ Petrosian magnitude versus effective $K$ s surface brightness for the $K \mathrm{~s}_{A B}<18.2, z<1$ sample selected in Fig. 9. The hard limit at the top-right hand side shows the size limit of $0.7^{\prime \prime}$. The main galaxy population seems to have a high surface brightness limit of $\mu_{K \mathrm{~s}}=18$. mag $\operatorname{arcsec}^{-2}$, with a small group at higher surface brightnesses (shown as blue crosses) - either compact galaxies or stars that have managed to avoid all the selection criteria. Galaxies have surface brightnesses as low as $\mu_{K \mathrm{~s}}=24.0 \mathrm{mag} \operatorname{arcsec}^{-2}$, but fainter galaxies would need to be selected before the VIKING surface brightness became a limiting factor.

\subsection{Extragalactic variables in VIDEO}

In deep extragalactic surveys, such as VIDEO, with many epochs over several months or years, it is possible to find a range of AGN, and very occasionally supernovae. There are also a few foreground stars that show variability. We select point-source variables in the VIDEO survey which show a range in magnitudes greater than $0.1 \mathrm{mag}$ in any filter. Since the filters in this survey are not taken simultaneously, and AGN show sporadic variability, sometimes variations may only be seen in one filter.

SELECT s.sourceID, s.ra,s.dec,v.frameSetID, v.zMedianMag, v.zMagRms, v.znGoodObs, v.zSkewness, (v.zMaxMag-v.zMinMag) AS zRange,v.yMedianMag, $\mathrm{v}$. yMagRms, v. ynGoodObs, v.ySkewness, (v.yMaxMag-v.yMinMag) AS yRange,v.jMedianMag, v. jMagRms ,v. jnGoodObs, v. jSkewness, (v.jMaxMag-v.jMinMag) AS jRange,v.hMedianMag, v.hMagRms, v.hnGoodObs, v.hSkewness,

(v.hMaxMag-v.hMinMag) AS hRange,v.ksMedianMag, v.ksMagRms, v.ksnGoodObs, v.ksSkewness,

(v.ksMaxMag-v.ksMinMag) AS ksRange

FROM videoVariability AS $\mathrm{v}$, videoSource AS

$\mathrm{s} / *$ join tables */

WHERE $v$. sourceID $=s$. sourceID AND

$/ *$ point source variables */

s.mergedClass IN $(-1,-2)$ AND

v. variableClass $=1$ AND

$/ *$ delta mag in $>0.1$ in ANY filter, with at least 5 good obs in that filter */

( ( (zMaxMag-zMinMag) $>0.1$ AND zMinMag $>0$.

AND znGoodObs $>=5)$ OR ((yMaxMag-yMinMag) $>0.1$

AND yMinMag $>0$. AND ynGoodObs $>=5)$ OR

( (jMaxMag-jMinMag) $>0.1$ AND jMinMag $>0$. AND

jnGoodObs $>=5)$ OR ((hMaxMag-hMinMag) $>0.1$

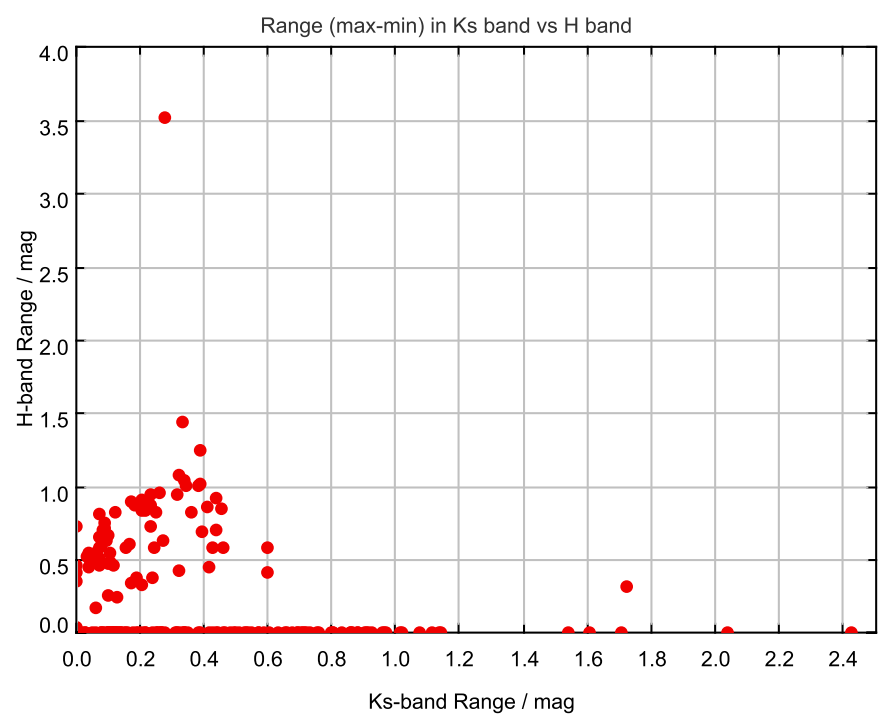

Fig. 12. $K$ s-band magnitude range versus $H$-band magnitude range. While there is a correlation between the two ranges, the $H$-band range seems greater than the $K \mathrm{~s}$-band on average. There are some objects which have no discernible variation in $K$ s or $H$, but do so in one or more of the other filters.

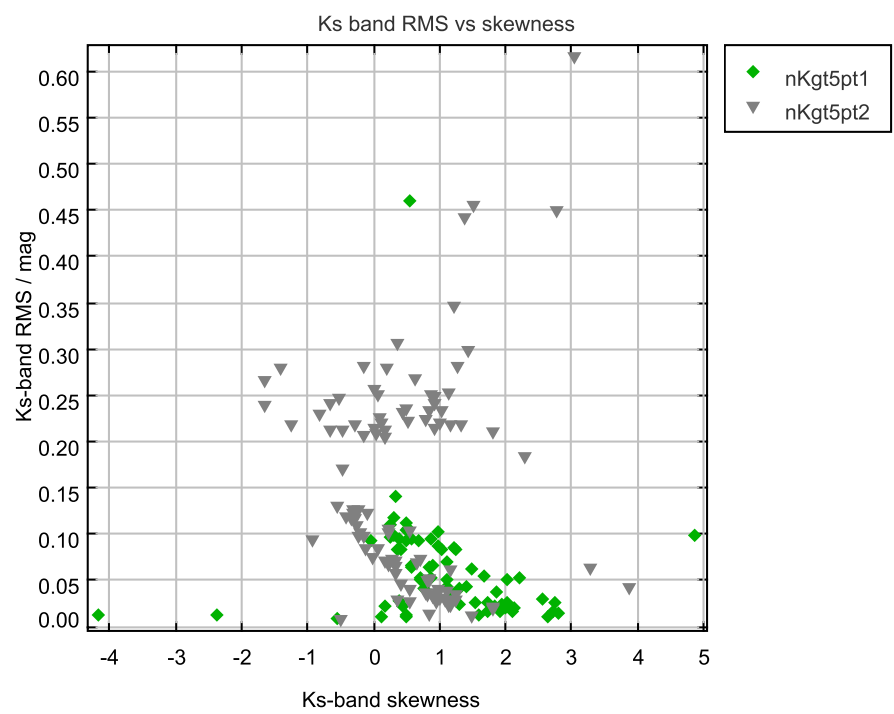

Fig. 13. $K$ s-band Skewness versus $\mathrm{rms}$ for objects with at least 5 good $K$ s-band observations. These have been split into the two pointings (pointing 1 , frameSetID $=644245094401$; pointing $2=$ 644245094402), which show similarities, at least for $\mathrm{rms}<0.2 \mathrm{mag}$. Most of the objects selected have positive skews in the Ks-band. The skew decreases as the RMS increases at rms $<0.2 \mathrm{mag}$, although the reason for this is not clear.

AND hMinMag $>0$. AND hnGoodObs $>=5$ ) OR ( (ksMaxMag-ksMinMag) $>0.1$ AND ksMinMag $>0$. AND ksnGoodObs $>=5$ ))

We can plot some of the variability statistics, such as the range in the $K_{\mathrm{S}}$ band against the range in the $H$ band, see Fig. 12 or the rms against the skewness in the $K_{\mathrm{s}}$ band, Fig. 13. These types of plots help to classify different types of variable and to pick out odd objects.

We can then select one of these objects, e.g. the object in Fig. 13 from pointing 1 , with a $K_{\mathrm{s}}$ band $\mathrm{rms}>0.4 \mathrm{mag}$, which 


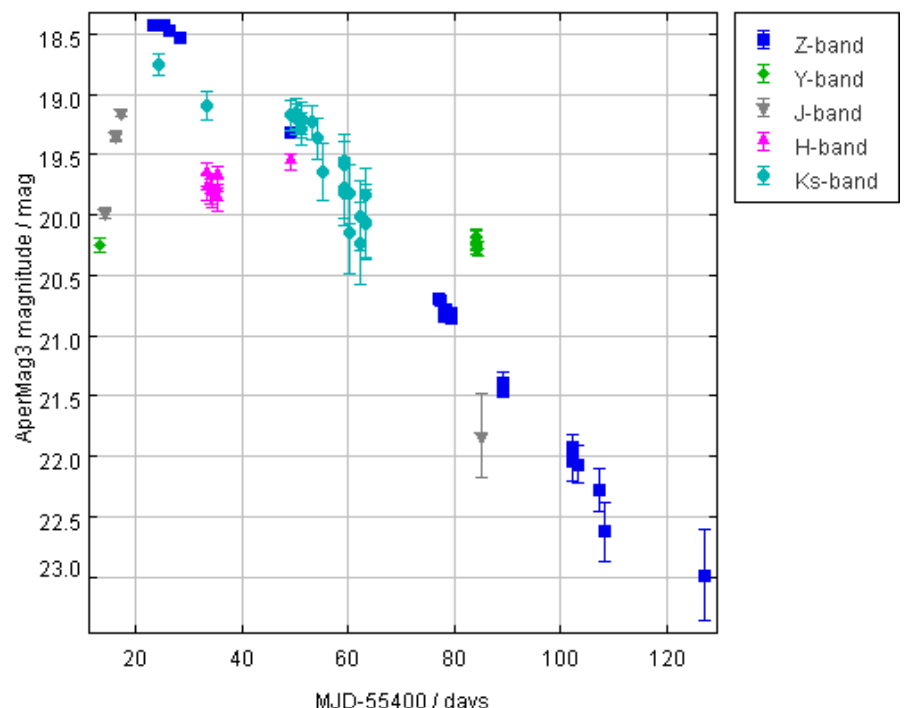

Fig. 14. Light-curve of variable selected in text. This variable brightens by $\sim 2$ mag in less than 10 days and then fades by almost 5 mag over the next 100 days. This is the expected behaviour of a supernova Type 1a.

is more than twice the RMS of any of the other objects and plot the light curve. To do this, we do a second query, below:

\section{SELECT}

/* Select time, filter, magnitude, magnitude error and flags */

d.mjd,d.filterID, d. aperMag3, d.aperMag3Err,

d.ppErrBits

/* From BestMatch table to link all

observations of the same source and

videoDetection for each observation */

FROM videoDetection as $d$,

videoSourceXDetectionBestMatch as b

/* First join tables */

WHERE b.multiframeID=d.multiframeID AND

b. extNum=d. extNum AND b. seqNum=d. seqNum

$/ *$ then select only detections and sourceID equal to object in previous selection

which has a Ks-band RMS $>0.4 \mathrm{mag} * /$

AND d.seqNum $>0$ AND b.sourceID IN (

SELECT S.SourceID

FROM videoVariability AS v, videoSource AS s $/ *$ join tables */

WHERE $\mathrm{v}$. sourceID $=\mathrm{s}$. sourceID AND

$/ *$ point source variables */

s.mergedClass IN $(-1,-2)$ AND

$\mathrm{v}$. variableClass $=1$ AND

$/$ * delta mag in $>0.1$ in ANY filter, with

at least 5 good obs in that filter */

( ( (zMaxMag-zMinMag) $>0.1$ AND zMinMag $>0$.

AND znGoodObs $>=5)$ OR ((yMaxMag-yMinMag) $>0.1$

AND yMinMag $>0$. AND ynGoodObs $>=5)$ OR

( $(j M a x M a g-j M i n M a g)>0.1$ AND jMinMag $>0$. AND

jnGoodObs $>=5)$ OR ((hMaxMag-hMinMag) $>0.1$ AND

hMinMag $>0$. AND hnGoodObs $>=5$ ) OR

$((\mathrm{ksMaxMag}-\mathrm{ksMinMag})>0.1$ AND ksMinMag $>0$. AND

ksnGoodObs $>=5$ ))

$/ *$ Ks-band RMS >0.4 mag */

AND ksMagRms $>0.4$ AND s.frameSetID=644245094401)

$/ *$ order by time */

ORDER BY d.mjd

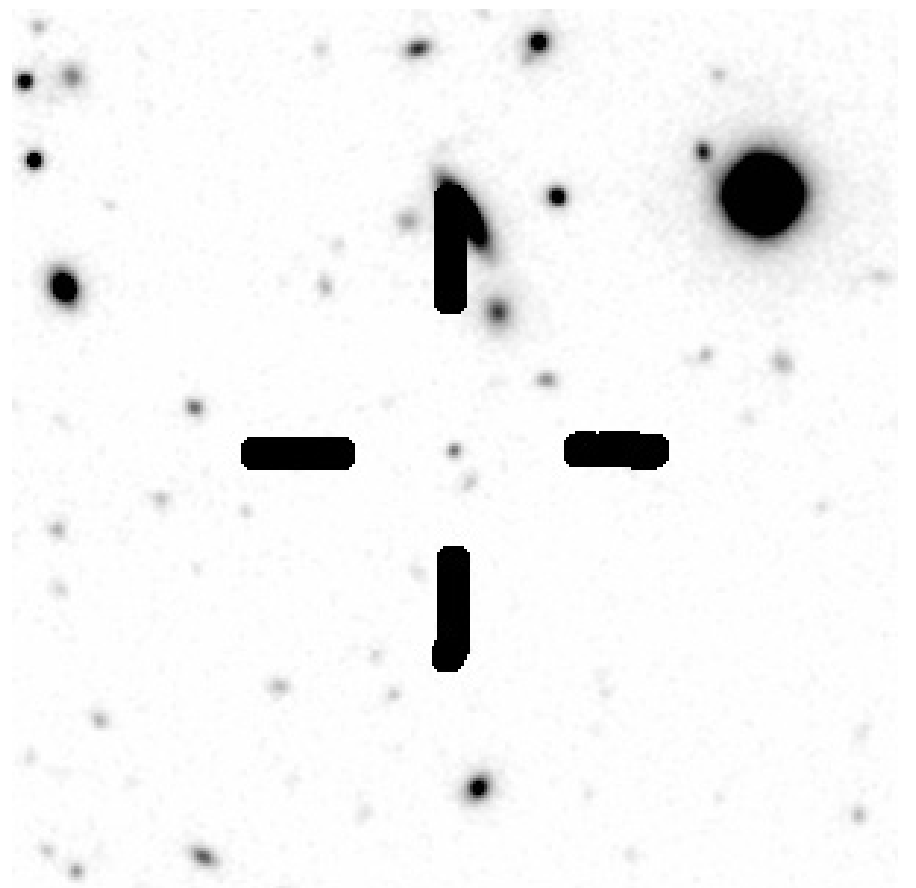

Fig. 15. Thumbnail of point-source variable which has light curve shown in Fig. 14. Thumbnails can be shown by selecting the attributes RA, Dec and frameSetID from the Source table and clicking on the supplied link.

We can use TOPCAT ${ }^{17}$ to plot the light-curve, see Fig. 14. The light-curve is very interesting, showing a short phase of brightening followed by a longer phase of fading, characteristic of an exploding star, probably a Type $1 \mathrm{a} \mathrm{SNa}$, with a maximum brightness of $Z=18.5 \mathrm{mag}$. The position and time of this object match SN2010gy (Chornock et al. 2010). The discovery team found that it has a redshift, $z=0.06$, but could not find a likely host galaxy. The thumbnail of this source from the deep $K_{\mathrm{s}}$ band mosaic is shown in Fig. 15.

\section{First public data releases}

The first public releases of VISTA Public Survey data through the VSA are intended to match the DR1/2 datasets published for each survey in the ESO SAF ${ }^{18}$. Thus, they will cover the data up to the end of ESO semester P85 (i.e. up to 30th September 2010). Some of the surveys have released data from P86 as well, but with the following additional constraints:

- VMC: the data released will only be those fields where the whole set of epochs is complete, i.e. the 30 Doradus field $\left(5^{\mathrm{h}} 37^{\mathrm{m}} 40^{\mathrm{s}},-69^{\circ} 22^{\prime} 18^{\prime \prime}\right)$ and the Gaia South Elliptical Pole field $\left(5^{\mathrm{h}} 59^{\mathrm{m}} 23^{\mathrm{s}},-66^{\circ} 20^{\prime} 28^{\prime \prime}\right)$.

- VIKING: the data will only be released in the following fields: GAMA09 (33 fields overlapping with the Galaxy and Mass Assembly 09h field; GAMA, Driver et al. 2011), CFHTLS-W1 (6 fields overlapping with the Canada France Hawaii Legacy Survey W1 field ${ }^{19}$ and 9 fields in the Southern Galactic Pole region.

\footnotetext{
17 http://www.star.bris.ac.uk/ mbt/topcat/

${ }^{18}$ http://www.eso.org/sci/observing/phase3/ data_releases.html

19 http://www.cfht.hawaii.edu/Science/CFHLS/
} 
Table 1. Summary of VISTA Public Survey DR1 VSA releases.

\begin{tabular}{lcccc}
\hline \hline Survey & $N$ (pointings) & $\begin{array}{c}\text { Filters and depth } \\
A B(\mathrm{mag})\end{array}$ & $\begin{array}{c}N(\text { epochs }) \\
\text { (typical) }\end{array}$ & $N$ (sources) \\
\hline VHS & 1260 & $Y \sim 20.9, J \sim 20.9, H \sim 20.7, K_{\mathrm{s}} \sim 20.2$ & $1,1,1,1$ & $1.2 \times 10^{8}$ \\
VVV & 350 & $Z \sim 21.9, Y \sim 20.9, J \sim 20.8, H \sim 20.2, K_{\mathrm{s}} \sim 19.3$ & $1,1,1,1,6$ & $5.0 \times 10^{8}$ \\
VMC & 2 & $Y \sim 21.9, J \sim 21.9, K_{\mathrm{s}} \sim 22.1$ & $4,4,12$ & $1.8 \times 10^{6}$ \\
VIKING & 48 & $Z \sim 23.0, Y \sim 21.9, J \sim 21.8, H \sim 21.4, K_{\mathrm{s}} \sim 21.3$ & $1,1,2,1,1$ & $5.3 \times 10^{6}$ \\
VIDEO & 2 & $Z \sim 25.6, Y \sim 25.1, J \sim 25.2, H \sim 24.8, K_{\mathrm{s}} \sim 24.5$ & $33,31,38,40,50$ & $7.5 \times 10^{5}$ \\
\hline
\end{tabular}

Notes. ${ }^{(a)}$ For multi-epoch filters, this is calculated from the catalogue, but for a single epoch, it is estimated from the exposure time.

- VIDEO: the data will only be released in the pointings which VIDEO mosaics have been created in (XMM3 field, $2^{\mathrm{h}} 26^{\mathrm{m}} 18^{\mathrm{s}},-4^{\circ} 44^{\prime} ;$ ES1-North field, $\left.0^{\mathrm{h}} 37^{\mathrm{m}} 49^{\mathrm{s}},-43^{\circ} 30^{\prime}\right)$.

We have cropped all the tables in the surveys to match the pointings specified by the PIs. The excluded data will be released in future releases. Table 1 summarises the contents of the releases. Unlike UKIDSS each survey will be released into a separate database. The sixth public survey UltraVISTA is not using VDFS processing, except for the initial pawprint pipeline calibration, so we are not releasing data from this survey.

\section{Summary and future work}

The VSA was designed as the main access point to all VISTA science data, allowing users to carefully select the data they need, rather than to bulk download all the data, a difficult and time consuming job in the era of billion row catalogues. As we have shown in the illustrated examples (Sect. 9), the VSA is designed to allow users to select on a wide range of attributes and to work with external data, such as the Sloan Digital Sky Survey, WISE, Glimpse, OGLE etc. The VSA is based on the WFCAM Science Archive but has VISTA specific features and various improvements based on our experience of WFCAM data and archive processing.

In the future we plan several enhancements. In the near future, we are working on improvements to our interface, including a MyDB (Li \& Thakar 2008) style access, where users can combine queries with Python scripts, to produce a powerful work environment. We are also improving our plotting tools to more easily show very large datasets with a combination of density maps where the number of points is huge, and individual points where the density drops below a threshold.

Acknowledgements. Many people have contributed to the development of the VISTA Science Archive. We would like to thank the PIs and members of the VISTA Public Survey teams who have made requests, spotted mistakes, and checked the early releases carefully. We would also like to thank users of the WFCAM Science Archive. We thank Steve Warren for providing Fig. 2. We would like to thank the anonymous referee for his/her careful reading of the text and useful suggestions that have improved this paper. We would also like to thank Pratika Dayal for pointing out the VISTA/VDFS jargon that we have got so used to using. We thank Microsoft also for providing software via the Microsoft Development Network Academic Alliance, and eGenix for providing a free, non-commercial licence for Python middleware. Financial resources for VISTA/WFCAM Science Archive development and operations were provided by the UK Science and Technology Facilities Council.

\section{Appendix A: Image scaling}

For single epoch OB stacks,

$$
\text { bscale }=\frac{1}{\text { NDIT } \sqrt{\text { NJITTER }}},
$$

and for tiles,

$$
\text { bscale }=\frac{1}{\text { NDIT } \sqrt{2 N J I T T E R}},
$$

where NDIT is the number of readouts during the integration of a raw image and NJITTER is the number of jitter positions in the jitter pattern to create a single pawprint stack. For deep stacks, we would scale by the number of epochs, in the same way as the number of jitters, but since NDIT and NJITTER can vary from stack to stack in the same programme, pointing and filter, we calculate bscale for deep stacks as:

$$
\text { bscale }^{\text {deep }}=\frac{1}{\sqrt{\sum_{i} \frac{1}{\text { bscale }_{i}^{2}}}} .
$$

For deep tiles, to take account of different integration times in each offset and deprecated detectors in some OB stacks, we compute the bscale values for each detector as above in each overlap and average over all overlaps, just as we calculate the total exposure time for a tile.

\section{Appendix B: Half-light radii}

The sizes of extended sources are difficult to measure for various reasons:

- The outer parts of a galaxy eventually merge into the sky background, so it is difficult to know how much of the galaxy is lost in the sky. For intrinsically low surface brightness galaxies or high redshift galaxies, the majority of the light may well be lost in the background and any measurement is a significant underestimate (e.g., Disney 1976; Cross et al. 2001)

- The profile is not always smooth or axisymmetrical, e.g. grand design spirals, irregular galaxies, interacting galaxies.

- Nearby objects can make it difficult to get accurate measurements of the total luminosity and extent of a galaxy. The measurement of the background level is sometimes incorrect and this can affect a curve-of-growth measurement.

- Galaxies have various inclinations to the line of sight. Different researchers may want to use measurements that correct for inclination or do not.

- The light of galaxies and all objects is convolved with a point-spread function that will particularly effect small objects.

- The method must be robust enough and quick enough to be applied to the VISTA detection tables. We exclude the VVV and VMC since these are in extremely dense regions where contamination from nearby objects is almost guaranteed and the vast majority of sources are stellar, which are point-sources. Even so, the catalogues with size measurements will contain $>10^{8}$ sources, and maybe $10^{9}$ sources. 
Our measurements of the size of galaxies try to take into account all the above effects as much as possible. We define our basic size measurement as the radius containing half of the flux of the galaxy - the half-light-radius - a measurement that has been used extensively before (Kormendy 1977; Cross et al. 2001; Blanton et al. 2001). The main difficulty with this measurement is measuring the total flux.

To take into account the missing light from the outer parts of the galaxy, we use the Petrosian flux (Blanton et al. 2001; Graham et al. 2005), which is generally insensitive to the effects of surface brightness, i.e. if you keep the galaxy profile the same (the relative flux as a function of radius) but reduce or increase the average surface-brightness, closer to or further from the sky noise value, then the Petrosian flux measurement will return the same flux each time. This breaks down eventually: if you reduce the surface brightness enough the galaxy won't even be detectable against the sky, and close to this limit the total flux and size will become difficult to measure with any accuracy.

The Petrosian flux however gives different results for different profiles, which is an issue. Blanton et al. (2001) showed that while only $0.7 \%$ of the flux of an exponential disk galaxy was typically missed by the Petrosian, $22 \%$ of the flux of a de Vaucouleurs' profile elliptical galaxy was missed, and $5 \%$ of the flux of a PSF dominated profile was missed, although Graham $\&$ Driver (2005) shows that there are slightly different results for a standard Petrosian definition compared to the SDSS Petrosian that Blanton used. Small galaxies, close to the seeing limit will be dominated by the point-spread function. Galaxies close to the surface-brightness limit of the survey could be missing much more of the light. To try to take into account the missing light, we assume that all galaxies are missing $10 \%$. This will be an overestimate in some cases and an underestimate in others, but to try and calculate a correction for each galaxy would require an iterative procedure which would take much longer, and in any case, it would be better to fit profiles for all objects (e.g. Peng et al. 2002). The light profiles of galaxies are often well fit by Sérsic profiles (Graham et al. 2005), a more general function, that includes exponential $(\beta=1)$, de Vaucouleur $(\beta=4)$ and Gaussian $(\beta=0.5)$,

$I(r)=I_{r_{\mathrm{hl}}} \exp \left\{-k\left[\left(\frac{r}{r_{\mathrm{hl}}}\right)^{1 / \beta}-1\right]\right\}$

where $r_{\mathrm{hl}}$ is the half-light radius, $\beta$ is the Sérsic index. To save time, we use the existing catalogue products to calculate the half-light radii, rather than going back to the images (e.g. Liske et al. 2003). The half-light radii are calculated using the existing circular aperture radii measurements of the flux, which give a curve-of-growth. We use the 13 aperture fluxes measured by the VDFS extractor, at radii of $0.5,0.5 \sqrt{2}, 1, \sqrt{2}, 2,2 \sqrt{2}, 4,5,6,7,8,10,12$ arcseconds. To calculate the half-light radius, we first find the aperture flux closest to half the total-flux and then use the five apertures centred on this ( 2 before, 2 after and the aperture in question). Using these 5 aperture fluxes, we fit a quadratic, which removes any small bumps in the curve, using the singular value decomposition method (Golub \& Reinsch 1970). We find the root of the quadratic that gives the half-light radius $\left(r_{\mathrm{hl}}^{\mathrm{c}}\right.$, hlCircRadAs). We use the covariance matrix to calculate the error in the half-light radius $\left(\sigma_{r_{h}^{c}}\right.$, hlCircRadErrAs), adding in another half-pixel in quadrature, to take into account the granularity of the data.

The 13 aperture fluxes are all circular apertures, so the halflight radius calculated assumes a circular symmetry. However, most galaxies are elliptical in shape, either being triaxial

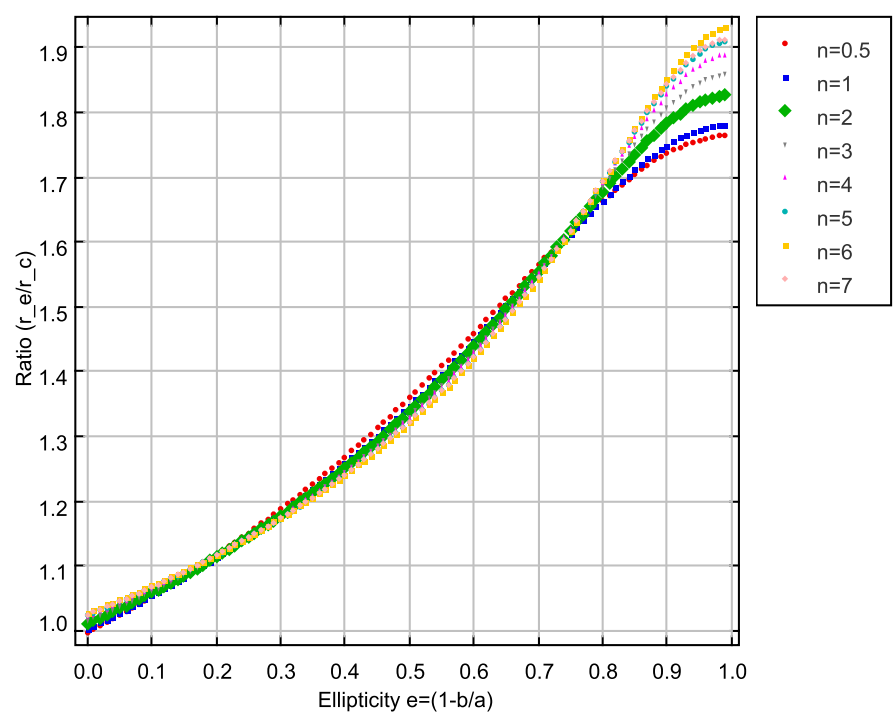

Fig. B.1. Ellipticity versus ratio of half-light semi-major axis to halflight radius for different Sérsic profiles. The lines are the best fit Moffat profiles in each case.

spheroidal systems or inclined disks or a combination of the two. A geometric mean size is usually considered a more useful measurement for triaxial elliptical galaxies (e.g. see Binney \& Merrifield 1998), and a semi-major axis size (Driver et al. 2005), which recovers the radius of the disk whatever the inclination, is more useful for disk galaxies. Figure B. 1 shows the ratio of halflight semi-major axis to half-light circular radius as a function of ellipticity for a range of Sérsic profiles $(\beta=0.5$ to $\beta=7)$ : profiles which are a good fit to the vast majority of virialised galaxies. As can be seen, the variation between these profiles is around $1-2 \%$ for all ellipticities $<0.9$, but rises to $10 \%$ at maximum ellipticity. These curves are well fit by Moffat functions,

$f(\epsilon)=\frac{a}{\left(1+\left(\frac{1-\epsilon}{b}\right)^{2}\right)^{c}}$

where $a, b \& c$ are found from fitting the data for each profile.

Thus, we can convert our circular half-light radii $r_{\mathrm{hl}}^{\mathrm{c}}$ to a semi-major axis size $r_{\mathrm{hl}}^{\mathrm{smj}}$ by using the $\beta=2$ function ( $a=$ $1.8243, b=0.30914, c=0.24304)$. We choose $\beta=2$, since most galaxies will be ellipticals $(\beta=1)$, de Vaucouleurs $(\beta=4)$, or dominated by the PSF ( $\beta=0.5)$, and $\beta=2$ is nicely in the middle, but as Fig. B. 1 shows, there is very little difference.

The conversions to the half-light semi-minor axis, $r_{\mathrm{hl}}^{\mathrm{smn}}$, and half-light geometric mean, $r_{\mathrm{hl}}^{\mathrm{geo}}$, are easily computed from the geometry of an ellipse:

$r_{\mathrm{hl}}^{\mathrm{smn}}=(1-\epsilon) r_{\mathrm{hl}}^{\mathrm{smj}}$
$r_{\mathrm{hl}}^{\mathrm{geo}}=\sqrt{r_{\mathrm{hl}}^{\mathrm{smn}} r_{\mathrm{hl}}^{\mathrm{smj}}}$.

Finally, we take into account the effects of seeing. We use the method of Driver et al. (2005) to subtract the measured seeing, assuming a Gaussian PSF,

$r_{\mathrm{hl}}^{\mathrm{smj}, \text { see }}=\sqrt{r_{\mathrm{hl}}^{\mathrm{smj}^{2}}-c_{\mathrm{see}} \Gamma^{2}}$,

where $\Gamma$ is the full-width half maximum of stars in the image and $c_{\text {see }}$ is a constant, 0.5 for a Gaussian PSF. By experiment we found values of $c_{\text {see }} \sim 0.45$, but with quite a large uncertainty, so we took the theoretical value $c_{\text {see }}=0.5$. 


\section{N. J. G. Cross et al.: The VISTA Science Archive}

\section{References}

Bertin, E., \& Arnouts, S. 1996, A\&AS, 117, 393

Binney, J., \& Merrifield, M. 1998, Galactic Astronomy

Blanton, M. R., Dalcanton, J., Eisenstein, D., et al. 2001, AJ, 121, 2358

Budavári, T., Szalay, A. S., \& Fekete, G. 2010, PASP, 122, 1375

Calabretta, M. R., \& Greisen, E. W. 2002, A\&A, 395, 1077

Casali, M., Adamson, A., Alves de Oliveira, C., et al. 2007, A\&A, 467, 777

Chornock, R., Huber, M., Foley, R. J., et al. 2010, Central Bureau Electronic Telegrams, 2414, 1

Cioni, M.-R. L., Clementini, G., Girardi, L., et al. 2011, A\&A, 527, A116

Collins, R., Cross, N., Sutorius, E., Read, M., \& Hambly, N. 2009, in Astronomical Data Analysis Software and Systems XVIII, eds. D. A. Bohlender, D. Durand, \& P. Dowler (San Francisco: ASP), 226

Collins, R., \& Hambly, N. 2012. Astronomical Data Analysis Software and Systems XXI, Proc. of a Conference held at Marriott Rive Gauche Conference Center, Paris, France, 6-10 November, 2011, eds. P. Ballester, D. Egret, \& N. P. F. Lorente (San Francisco: ASP), ASP Conf. Ser., 461, 525

Cross, N., Collins, R., Sutorius, E., et al. 2011, in Astronomical Data Analysis Software and Systems XX, eds. I. N. Evans, A. Accomazzi, D. J. Mink, \& A. H. Rots, ASP Conf. Ser., 442, 45

Cross, N. J. G., Driver, S. P., Couch, W., et al. 2001, MNRAS, 324, 825

Cross, N. J. G., Collins, R. S., Hambly, N. C., et al. 2009, MNRAS, 399, 1730

Dalton, G. B., Sutherland, W. J., Emerson, J. P., et al. 2010, in SPIE Conf. Ser., 7735

Disney, M. J. 1976, Nature, 263, 573

Driver, S. P., Liske, J., Cross, N. J. G., De Propris, R., \& Allen, P. D. 2005, MNRAS, 360, 81

Driver, S. P., Hill, D. T., Kelvin, L. S., et al. 2011, MNRAS, 413, 971

Emerson, J., \& Sutherland, W. 2010a, The Messenger, 139, 2

Emerson, J. P., \& Sutherland, W. J. 2010b, in SPIE Conf. Ser., 7733

Emerson, J. P., Irwin, M. J., Lewis, J., et al. 2004, in SPIE Conf. Ser., 5493, eds. P. J. Quinn, \& A. Bridger, 401
Emerson, J., McPherson, A., \& Sutherland, W. 2006, The Messenger, 126, 41 Findlay, J. R., Sutherland, W. J., Venemans, B. P., et al. 2012, MNRAS, 419, 3354

Fruchter, A. S., \& Hook, R. N. 2002, PASP, 114, 144

Golub, G., \& Reinsch, C. 1970, Numerische Mathematik, 14, 403

Graham, A. W., \& Driver, S. P. 2005, PASA, 22, 118

Graham, A. W., Driver, S. P., Petrosian, V., et al. 2005, AJ, 130, 1535

Hambly, N. C., Collins, R. S., Cross, N. J. G., \& et al. 2008, MNRAS, 384, 637

Irwin, M. 2009, UKIRT Newsletter, 25, 15

Irwin, M. 2010, UKIRT Newsletter, 26, 14

Irwin, M. J., Lewis, J., Hodgkin, S., et al. 2004, in SPIE Conf. Ser., 5493, eds. P. J. Quinn, \& A. Bridger, 411

Jarvis, M. J., Bonfield, D. G., Bruce, V. A., et al. 2012, MNRAS, accepted

Kormendy, J. 1977, ApJ, 218, 333

Lawrence, A., Warren, S. J., Almaini, O., et al. 2007, MNRAS, 379, 1599

Lewis, J. R., Irwin, M., \& Bunclark, P. 2010, in Astronomical Data Analysis Software and Systems XIX, eds. Y. Mizumoto, K.-I. Morita, \& M. Ohishi, ASP Conf. Ser., 434, 91

Li, N., \& Thakar, A. R. 2008, Comput. Sci. Engin., 10, 18

Liske, J., Lemon, D. J., Driver, S. P., Cross, N. J. G., \& Couch, W. J. 2003 MNRAS, 344, 307

McCracken, H. J., Milvang-Jensen, B., Dunlop, J., et al. 2012, A\&A, 544, A156

Pence, W. D., Chiappetti, L., Page, C. G., Shaw, R. A., \& Stobie, E. 2010, A\&A, $524, \mathrm{~A} 42$

Peng, C. Y., Ho, L. C., Impey, C. D., \& Rix, H.-W. 2002, AJ, 124, 266

Saito, R. K., Hempel, M., Minniti, D., et al. 2012, A\&A, 537, A107

Skrutskie, M. F., Cutri, R. M., Stiening, R., \& et al. 2006, AJ, 131, 1163

Smith, A. J., Loveday, J., \& Cross, N. J. G. 2009, MNRAS, 397, 868

Szalay, A. S., Gray, J., Thakar, A. R., et al. 2002 [arXiv:cs/0202013]

Taylor, M. B. 2005, in Astronomical Data Analysis Software and Systems XIV, eds. P. Shopbell, M. Britton, \& R. Ebert, ASP Conf. Ser., 347, 29

York, D. G., Adelman, J., Anderson, Jr., J. E., et al. 2000, AJ, 120, 1579 